\title{
Irreducible holonomy algebras of Riemannian supermanifolds
}

\author{
Anton S. Galaev
}

November 11, 2018

\begin{abstract}
Possible irreducible holonomy algebras $\mathfrak{g} \subset \mathfrak{o s p}(p, q \mid 2 m)$ of Riemannian supermanifolds under the assumption that $\mathfrak{g}$ is a direct sum of simple Lie superalgebras of classical type and possibly of a one-dimensional center are classified. This generalizes the classical result of Marcel Berger about the classification of irreducible holonomy algebras of pseudoRiemannian manifolds.

Keywords: Riemannian supermanifold, Levi-Civita superconnection, holonomy algebra, Berger superalgebra
\end{abstract}

\section{Contents}

1 Introduction $\quad 2$

2 Holonomy algebras of pseudo-Riemannian manifolds 3

\begin{tabular}{lll}
3 & Lie superalgebras & 5 \\
\hline
\end{tabular}

4 Supermanifolds and their holonomy groups $\quad 7$

$\begin{array}{lll}5 & \text { Berger superalgebras } & 9\end{array}$

6 The Main Results $\quad 10$

$\begin{array}{llr}7 & \text { Weak-Berger algebras } & 12\end{array}$

8 The case of Riemannian odd supermanifolds $\quad 12$

9 Structure of the spaces $\mathcal{R}(\mathfrak{g}) \quad[14$

10 Adjoint representations of simple Lie superalgebras 17

11 Proof of Theorem 2 


\section{Introduction}

Possible irreducible holonomy algebras (or, equivalently, connected irreducible holonomy groups) of non-locally symmetric pseudo-Riemannian manifolds are classified by $\mathrm{M}$. Berger in [4]. These algebras are the following: $\mathfrak{s o}(p, q), \mathfrak{s o}(p, \mathbb{C}), \mathfrak{u}(r, s), \mathfrak{s u}(r, s), \mathfrak{s p}(r, s), \mathfrak{s p}(r, s) \oplus \mathfrak{s p}(1)$, $\mathfrak{s p}(r, \mathbb{R}) \oplus \mathfrak{s l}(2, \mathbb{R}), \mathfrak{s p}(r, \mathbb{C}) \oplus \mathfrak{s l}(2, \mathbb{C}), \mathfrak{s p i n}(7) \subset \mathfrak{s o}(8), \mathfrak{s p i n}(4,3) \subset(4,4), \mathfrak{s p i n}(7, \mathbb{C}) \subset \mathfrak{s o}(8,8)$, $G_{2} \subset \mathfrak{s o}(7), G_{2(2)}^{*} \subset \mathfrak{s o}(4,3)$ and $G_{2}^{\mathbb{C}} \subset \mathfrak{s o}(7,7)$. This result, especially in the case of Riemannian manifolds, has a lot of consequences and applications both in geometry and physics, see [2, 6, 8, 21] and the references therein.

Since theoretical physicists discovered supersymmetry, supermanifolds began to play an important role both in mathematics and physics [11, 26, 28, 39]. There is also an interest to Riemannian supermanifolds, see e.g. [3, 7, 9, 10, 18. In particular, Calabi-Yau supermanifolds (i.e. Riemannian supermanifolds with the holonomy algebras contained in $\mathfrak{s u}\left(p_{0}, q_{0} \mid p_{1}, q_{1}\right)$ ) were considered recently in several physical papers, e.g. [1, 33, 41].

Holonomy groups and holonomy algebras for superconnections on supermanifolds are introduced recently in [15. In the present paper we generalize the result of M. Berger for the case of Riemannian supermanifolds.

For a supersubalgebra $\mathfrak{g} \subset \mathfrak{o s p}(p, q \mid 2 m)$ we define the vector superspace $\mathcal{R}(\mathfrak{g})$ of curvature tensors of type $\mathfrak{g}$ that consists of linear maps from $\Lambda^{2} \mathbb{R}^{p, q \mid 2 m}$ to $\mathfrak{g}$ satisfying the Bianchi super identity. We call $\mathfrak{g}$ a Berger superalgebra if $\mathfrak{g}$ is spanned by the images of the elements of $\mathcal{R}(\mathfrak{g})$. The holonomy algebra of a Riemannian supermanifold is a Berger superalgebra. Consequently, Berger superalgebras may be considered as the candidates to the holonomy algebras. A Berger superalgebra $\mathfrak{g} \subset \mathfrak{o s p}(p, q \mid 2 m)$ is called symmetric if the superspace $\mathcal{R}^{\nabla}(\mathfrak{g})$, consisting of linear maps from $\mathbb{R}^{p, q \mid 2 m}$ to $\mathcal{R}(\mathfrak{g})$ satisfying the second Bianchi super identity, is trivial. If such $\mathfrak{g}$ is the holonomy algebra of a Riemannian supermanifold, then this supermanifold is locally symmetric. The classification of irreducible symmetric Berger superalgebras can be deduced from [38]. Irreducible Berger supersubalgebras $\mathfrak{g} \subset \mathfrak{o} \mathfrak{s p}(0 \mid 2 m)$ are classified in [17]. In the present paper we classify irreducible non-symmetric Berger supersubalgebras $\mathfrak{g} \subset \mathfrak{o s p}(p, q \mid 2 m)$ $(p+q>0)$ of the form

$$
\mathfrak{g}=\left(\oplus_{i} \mathfrak{g}_{i}\right) \oplus \mathfrak{z},
$$

where $\mathfrak{g}_{i}$ are simple Lie superalgebras of classical type and $\mathfrak{z}$ is a trivial or one-dimensional center. The obtained list is the following:

$$
\begin{aligned}
& \mathfrak{o s p}(p, q \mid 2 m), \quad \mathfrak{o} \mathfrak{s p}(r \mid 2 k, \mathbb{C}), \quad \mathfrak{u}\left(p_{0}, q_{0} \mid p_{1}, q_{1}\right), \quad \mathfrak{s u}\left(p_{0}, q_{0} \mid p_{1}, q_{1}\right), \\
& \mathfrak{h o s p}(r, s \mid k), \quad \mathfrak{h o s p}(r, s \mid k) \oplus \mathfrak{s p}(1), \quad \mathfrak{o s p}^{s k}(2 k \mid r, s) \oplus \mathfrak{s l}(2, \mathbb{R}), \\
& \mathfrak{o s p p}^{s k}(2 k \mid r, \mathbb{C}) \oplus \mathfrak{s l}(2, \mathbb{C}) .
\end{aligned}
$$

This list generalizes the list of irreducible holonomy algebras of non-locally symmetric pseudoRiemannian manifolds, in the same time, we do not get the analogs of the important holonomy algebras $G_{2}$ and $\mathfrak{s p i n}(7)$. We discuss the geometries of the supermanifolds with each of the obtained holonomy algebras.

Remark that in general a semi-simple Lie superalgebra $\mathfrak{g}$ has the form $\mathfrak{g}=\oplus_{i}\left(\mathfrak{g}_{i} \otimes \Lambda\left(n_{i}\right)\right)$, where $\mathfrak{g}_{i}$ is a simple Lie superalgebra (either of classical or of Cartan type), and $\Lambda\left(n_{i}\right)$ is the Grassmann superalgebra of $n_{i}$ variables [23]. Moreover, there exist representations of solvable

Lie superalgebras in vector superspaces of dimensions greater then one [23]. Thus we consider only a part of irreducible subalgebras $\mathfrak{g} \subset \mathfrak{o} \mathfrak{s}(p, q \mid 2 m)$. For irreducible subalgebras $\mathfrak{g} \subset \mathfrak{s o}(p, q)$ the property (1) holds automatically, i.e. our assumption is quit natural.

In Section 2 we recall some facts about holonomy algebras of pseudo-Riemannian manifolds. In Section 3 and 4 we provide some preliminaries on Lie superalgebras and supermanifolds. In Section 6 we formulate the main result of the paper. The remaining sections are dedicated to 
the proof. In Section 9 we describe the superspaces $\mathcal{R}(\mathfrak{g})$ for subalgebras $\mathfrak{g} \subset \mathfrak{o s p}(p, q \mid 2 m)$. Let $V=\mathbb{R}^{p, q \mid 2 m}$. We show that any element $R \in \mathcal{R}(\mathfrak{g})_{\overline{0}}$ satisfies

$$
\left.\operatorname{pr}_{\mathfrak{s o}(p, q)} \circ R\right|_{\Lambda^{2} V_{\overline{0}}} \in \mathcal{R}\left(\operatorname{pr}_{\mathfrak{s o}(p, q)} \mathfrak{g}_{\overline{0}}\right),\left.\quad \operatorname{pr}_{\mathfrak{s p}(2 m, \mathbb{R})} \circ R\right|_{\Lambda^{2} V_{\overline{1}}} \in \overline{\mathcal{R}}\left(\operatorname{pr}_{\mathfrak{s p}(2 m, \mathbb{R})} \mathfrak{g}_{\overline{0}}\right),
$$

where $\mathcal{R}\left(\operatorname{pr}_{\mathfrak{s o}(p, q)} \mathfrak{g}_{\overline{0}}\right)$ is the space of curvature tensors for the subalgebra $\operatorname{pr}_{\mathfrak{s o}(p, q)} \mathfrak{g}_{\overline{0}} \subset \mathfrak{s o}(p, q)$ and $\bar{R}\left(\operatorname{pr}_{\mathfrak{s p}(2 m, \mathbb{R})} \mathfrak{g}_{\overline{0}}\right)$ is a similar space for the subalgebra $\operatorname{pr}_{\mathfrak{s p}(2 m, \mathbb{R})} \mathfrak{g}_{\overline{0}} \subset \mathfrak{s p}(2 m, \mathbb{R})$, this space is defined in Section 8. Next, any $R \in \mathcal{R}(\mathfrak{g})_{\overline{1}}$ satisfies

$$
\left.\operatorname{pr}_{\mathfrak{s o}(p, q)} \circ R(\cdot, \xi)\right|_{V_{\overline{0}}} \in \mathcal{P}_{\eta}\left(\operatorname{pr}_{\mathfrak{s o}(p, q)} \mathfrak{g}_{\overline{0}}\right),\left.\quad \operatorname{pr}_{\mathfrak{s p}(2 m, \mathbb{R})} \circ R(\cdot, x)\right|_{V_{\overline{1}}} \in P_{\omega}\left(\operatorname{pr}_{\mathfrak{s p}(2 m, \mathbb{R})} \mathfrak{g}_{\overline{0}}\right)
$$

where $x \in V_{\overline{0}}$ and $\xi \in V_{\overline{1}}$ are fixed, $\mathcal{P}_{\eta}\left(\operatorname{pr}_{\mathfrak{s o}(p, q)} \mathfrak{g}_{\overline{0}}\right)$ is the space of weak curvature tensors for the subalgebra $\operatorname{pr}_{\mathfrak{s o}(p, q)} \mathfrak{g}_{\overline{0}} \subset \mathfrak{s o}(p, q)$ and $\mathcal{P}_{\omega}\left(\operatorname{pr}_{\mathfrak{s p}(2 m, \mathbb{R})} \mathfrak{g}_{\overline{0}}\right)$ is a similar space for the subalgebra $\operatorname{pr}_{\mathfrak{s p}(2 m, \mathbb{R})} \mathfrak{g}_{\overline{0}} \subset \mathfrak{s p}(2 m, \mathbb{R})$. We discuss these spaces in Sections 7 and 8. Elements of the space $\mathcal{P}_{\eta}(\mathfrak{s o}(n))$ appear as a part of the curvature tensor of a Lorentzian manifold [25, 14]. These properties of the space $\mathcal{R}(\mathfrak{g})$ give strong conditions on the representation $\mathfrak{g}_{\overline{0}} \subset \mathfrak{s} \mathfrak{o}(p, q) \oplus$ $\mathfrak{s p}(2 m, \mathbb{R})$ of the even part of $\mathfrak{g}$. We prove that under some assumption $\operatorname{pr}_{\mathfrak{s o}(p, q)} \mathfrak{g}_{\overline{0}} \subset \mathfrak{s o}(p, q)$ is the holonomy algebra of a pseudo-Riemannian manifold and $\operatorname{pr}_{\mathfrak{s p}(2 m, \mathbb{R})} \mathfrak{g}_{\overline{0}} \subset \mathfrak{s p}(2 m, \mathbb{R})$ is in the list of possible reductive holonomy algebras of Riemannian odd supermanifolds. These facts allow us to prove the classification theorem. Many facts and tables that we use are collected in [17.

\section{Holonomy algebras of pseudo-Riemannian manifolds}

The theory of holonomy algebras of pseudo-Riemannian manifolds can be found e.g. in [6, 8, 21$]$. Here we collect some facts that motivate the topic of this paper and will be used below.

Let $(M, g)$ be a connected pseudo-Riemannian manifold of signature $(p, q)$. The holonomy group of $(M, g)$ at a point $x \in M$ is a Lie group that consists of the pseudo-orthogonal transformations given by the parallel displacements along piece-wise smooth loops at the point $x$, and it can be identified with a Lie subgroup of the pseudo-orthogonal Lie group $\mathrm{O}(p, q)$. The corresponding Lie subalgebra of $\mathfrak{s o}(p, q)$ is called the holonomy algebras. If the manifold $M$ is simply connected, then the holonomy group is connected and it is uniquely defined by the holonomy algebra.

A subalgebra $\mathfrak{g} \subset \mathfrak{s o}(p, q)$ is called weakly-irreducible if it does not preserve any proper nondegenerate subspace of the pseudo-Euclidean space $\mathbb{R}^{p, q}$. By the $\mathrm{Wu}$ Theorem [40] any pseudoRiemannian manifold can be decomposed (at least locally) in the product of a flat pseudoRiemannian manifold and of pseudo-Riemannian manifolds with irreducible holonomy algebra. In particular, for any holonomy algebra $\mathfrak{g} \subset \mathfrak{s o}(V)$ there exists an orthogonal decomposition

$$
\mathbb{R}^{p, q}=V_{0} \oplus V_{1} \oplus \cdots \oplus V_{r}
$$

into a direct sum of pseudo-Euclidean subspaces and a decomposition

$$
\mathfrak{g}=\mathfrak{g}_{1} \oplus \cdots \oplus \mathfrak{g}_{r}
$$

into a direct sum of ideals such that $\mathfrak{g}_{i}$ annihilates $V_{j}$ if $i \neq j$ and $\mathfrak{g}_{i} \subset \mathfrak{s o}\left(V_{i}\right)$ is the weaklyirreducible holonomy algebra of a pseudo-Riemannian manifold.

Possible connected irreducible holonomy groups (i.e. possible irreducible holonomy algebras) of not locally symmetric pseudo-Riemannian manifolds classified Marcel Berger in [4]. Later it was proved that all these algebras can be realized as the holonomy algebras of pseudo-Riemannian manifolds [8]. Here is the list and the description of the corresponding geometries (see e.g. [8]): 
$\mathfrak{s o}(p, q)$ : generic pseudo-Riemannian manifolds;

$\mathfrak{s o}(p, \mathbb{C}) \subset \mathfrak{s o}(p, p)$ generic holomorphic pseudo-Riemannian manifolds;

$\mathfrak{u}(r, s) \subset \mathfrak{s o}(2 r, 2 s):$ pseudo-Kählerian manifolds;

$\mathfrak{s u}(r, s) \subset \mathfrak{s o}(2 r, 2 s)$ : special pseudo-Kählerian manifolds or Calabi-Yau manifolds;

$\mathfrak{s p}(r, s) \subset \mathfrak{s o}(4 r, 4 s):$ pseudo-hyper-Kählerian manifolds;

$\mathfrak{s p}(r, s) \oplus \mathfrak{s p}(1) \subset \mathfrak{s o}(4 r, 4 s):$ pseudo-quaternionic-Kählerian manifolds;

$\mathfrak{s p}(r, \mathbb{R}) \oplus \mathfrak{s l}(2, \mathbb{R}) \subset \mathfrak{s o}(2 r, 2 r)$ : pseudo-para-Kählerian manifolds;

$\mathfrak{s p}(r, \mathbb{C}) \oplus \mathfrak{s l}(2, \mathbb{C}) \subset \mathfrak{s o}(4 r, 4 r):$ complex pseudo-para-Kählerian manifolds;

$\mathfrak{s p i n}(7) \subset \mathfrak{s o}(8), \mathfrak{s p i n}(4,3) \subset \mathfrak{s o}(4,4), \mathfrak{s p i n}(7, \mathbb{C}) \subset \mathfrak{s o}(8,8):$ 8-dimensional pseudoRiemannian manifolds with a parallel 4 -form and their complex analog;

$G_{2} \subset \mathfrak{s o}(7), G_{2(2)}^{*} \subset \mathfrak{s o}(4,3), G_{2}^{\mathbb{C}} \subset \mathfrak{s o}(7,7):$ 7-dimensional pseudo-Riemannian manifolds with a parallel 3 -form and their complex analog.

Compact Riemannian manifolds with the holonomy groups $\mathrm{SU}(2), \mathrm{SU}(3), G_{2}$ and $\mathrm{Spin}(7)$ are extremely useful in theoretical physics, see references in [21].

The list of weakly-irreducible (and irreducible) reductive holonomy algebras of locally symmetric pseud-Riemannian manifolds can be found in [5].

In the case of Riemannian manifolds weakly-irreducible subalgebras $\mathfrak{g} \subset \mathfrak{s o}(n)$ are the same as the irreducible ones. Weakly-irreducible not irreducible Berger subalgebras $\mathfrak{g} \subset \mathfrak{s o}(p, q)$ are classified only if $p=1$ ( $p$ is the number of minuses of the metric), i.e. in the case of Lorentzian manifolds, if $\mathfrak{g} \subset \mathfrak{u}(1, q) \subset \mathfrak{s o}(2,2 q)$ and there are some partial results in the neural signature $(p, p)$, see the review [12].

Let us shortly explain how a classification of holonomy algebras can be obtained.

Let $\mathfrak{g} \subset \mathfrak{s o}(p, q)=\mathfrak{s o}(V)$ be a subalgebra, where $V=\mathbb{R}^{p, q}$ is the pseudo-Euclidean space. The space of curvature tensors of type $\mathfrak{g}$ is defined as follows

$$
\mathcal{R}(\mathfrak{g})=\left\{\begin{array}{c|c}
R \in \Lambda^{2} V^{*} \otimes \mathfrak{g} & \begin{array}{c}
R(X, Y) Z+R(Y, Z) X+R(Z, X) Y=0 \\
\text { for all } X, Y, Z \in V
\end{array}
\end{array}\right\} .
$$

The above identity is called the first Bianchi identity. Let $L(\mathcal{R}(\mathfrak{g})) \subset \mathfrak{g}$ be the ideal spanned by the images of the elements form $\mathcal{R}(\mathfrak{g})$. From the Ambrose-Singer Theorem it follows that for the holonomy algebra $\mathfrak{g}$ of a pseudo-Riemannian manifold it holds $L(\mathcal{R}(\mathfrak{g}))=\mathfrak{g}$.

Consider the vector space

$$
\mathcal{R}^{\nabla}(\mathfrak{g})=\left\{\begin{array}{l|l}
S \in V^{*} \otimes \mathcal{R}(g) & \begin{array}{c}
S_{X}(Y, Z)+S_{Y}(Z, X)+S_{Z}(X, Y)=0 \\
\text { for all } X, Y, Z \in V
\end{array}
\end{array}\right\} .
$$

If $\mathfrak{g}$ satisfies $\mathcal{R}^{\nabla}(\mathfrak{g})=0$, then any pseudo-Riemannian manifold with the holonomy algebra $\mathfrak{g}$ is automatically locally flat. M. Berger classified irreducible subalgebras $\mathfrak{g} \subset \mathfrak{s o}(p, q)$ that satisfy $L(\mathcal{R}(\mathfrak{g}))=\mathfrak{g}$ and $\mathcal{R}^{\nabla}(\mathfrak{g}) \neq 0$. By this reason subalgebra $\mathfrak{g} \subset \mathfrak{s o}(p, q)$ satisfying $L(\mathcal{R}(\mathfrak{g}))=\mathfrak{g}$ are called Berger algebras [29, 34]. If $\mathfrak{g} \subset \mathfrak{s o}(V)$ is a Berger subalgebra and $\mathcal{R}^{\nabla}(\mathfrak{g})=0$, then $\mathfrak{g}$ is called a symmetric Berger algebra, otherwise $\mathfrak{g}$ is called a non-symmetric Berger algebra. It is known that if $\mathfrak{g}$ is a reductive Lie algebra and the $\mathfrak{g}$-module $\mathcal{R}(\mathfrak{g})$ is trivial, then $\mathcal{R}^{\nabla}(\mathfrak{g})=0$ 34 . 
Let $\mathfrak{g} \subset \mathfrak{s o}(V)$ be a reductive weakly-irreducible Berger subalgebra. If $\mathfrak{g}$ is not irreducible, then it preserves a degenerate subspace $W \subset V$. Consequently, $\mathfrak{g}$ preserves the isotropic subspace $L=W \cap W^{\perp}$. Since $\mathfrak{g}$ is totally reducible, there exists a complementary invariant subspace $L^{\prime} \subset V$. Since $\mathfrak{g}$ is weakly-irreducible, the subspace $L^{\prime}$ is degenerate. If $L^{\prime}$ is not isotropic, then $\mathfrak{g}$ preserves the kernel of the restriction of the metric to $L^{\prime}$ and $\mathfrak{g}$ preserves a complementary subspace in $L^{\prime}$ to this kernel, which is non-degenerate. Hence $L^{\prime}$ is isotropic and $V=L \oplus L^{\prime}$ is the direct sum of isotropic subspaces. This can happen only if $p=q$. The metric on $V$ allows to identify $L^{\prime}$ with the dual space $L^{*}$ and the representations of $\mathfrak{g}$ on $L$ and $L^{\prime}$ are dual. This shows that the representation $\mathfrak{g} \subset \mathfrak{g l}(L)$ is irreducible. Let $R \in \mathcal{R}(\mathfrak{g})$. From the Bianchi identity it follows that $R(x, y)=0$ and $R(\varphi, \psi)=0$ for all $x, y \in L$ and $\varphi, \psi \in L^{*}$. Moreover, for each fixed $\varphi \in L^{*}$ it holds $R(\cdot, \varphi) \in(\mathfrak{g} \subset \mathfrak{g l}(L))^{(1)}$, where $(\mathfrak{g} \subset \mathfrak{g l}(L))^{(1)}$ is the first prolongation for the representation $\mathfrak{g} \subset \mathfrak{g l}(L)$ (similarly, for each fixed $x \in L$ it holds $\left.R(\cdot, x) \in\left(\mathfrak{g} \subset \mathfrak{g l}\left(L^{*}\right)\right)^{(1)}\right)$. Consequently, $(\mathfrak{g} \subset \mathfrak{g l}(L))^{(1)} \neq 0$. The list of irreducible subalgebras and $\mathfrak{g} \subset \mathfrak{g l}(L)$ with $(\mathfrak{g} \subset \mathfrak{g l}(L))^{(1)} \neq 0$ can be found in [8].

\section{$3 \quad$ Lie superalgebras}

Here we define some Lie superalgebras that we will use. Further information about Lie superalgebras can be found e.g. in [11, 23, 26]. For the purposes of this paper, in [17] we list simple Lie superalgebras of classical type.

$A$ vector superspace $V$ is a $\mathbb{Z}_{2}$-graded vector space $V=V_{\overline{0}} \oplus V_{\overline{1}}, \mathbb{Z}_{2}=\{\overline{0}, \overline{1}\}$. The elements $x \in V_{\overline{0}} \cup V_{\overline{1}}$ are called homogeneous. Elements $x \in V_{\overline{0}}$ are called even, and we define the parity $|x|$ of $x$ by putting $|x|=\overline{0}$, while elements $x \in V_{\overline{1}} \backslash\{0\}$ are called odd, we write $|x|=\overline{1}$. All notions and constructions defined for the usual vector spaces can be extended to the vector superspaces, i.g. if $V$ and $W$ are vector superspaces, then their tensor product $V \otimes W$ is a vector superspace with the grading

$$
(V \otimes W)_{\overline{0}}=\left(V_{\overline{0}} \otimes W_{\overline{0}}\right) \oplus\left(V_{\overline{1}} \otimes W_{\overline{1}}\right), \quad(V \otimes W)_{\overline{1}}=\left(V_{\overline{0}} \otimes W_{\overline{1}}\right) \oplus\left(V_{\overline{1}} \otimes W_{\overline{0}}\right) .
$$

Linear maps $F: V \rightarrow W$ that preserve the parity are called morphisms.

$A$ superalgebra is a vector superspace $A=A_{\overline{0}} \oplus A_{\overline{1}}$ with a morphism $\cdot: A \otimes A \rightarrow A$, i.e. for homogeneous vectors it holds $|x \cdot y|=|x|+|y|$. An important example of a superalgebra is a Grassmann superalgebra

$$
\Lambda(m)=\Lambda \mathbb{R}^{m}=\oplus_{k=1}^{m} \Lambda^{k} \mathbb{R}^{m}
$$

with the obvious $\mathbb{Z}_{2}$-grading. The Grassmann superalgebra is super commutative, this means that its homogeneous elements satisfy $x y=(-1)^{|x||y|} y x$.

A Lie superalgebra is a superalgebra $\mathfrak{g}=\mathfrak{g}_{\overline{0}} \oplus \mathfrak{g}_{\overline{1}}$ with the multiplication $[\cdot, \cdot]: \mathfrak{g} \otimes \mathfrak{g} \rightarrow \mathfrak{g}$ such that the homogeneous elements satisfy

$$
\begin{gathered}
{[x, y]=-(-1)^{|x||y|}[y, x],} \\
{[[x, y], z]+(-1)^{|x|(|y|+|z|)}[[y, z], x]+(-1)^{|z|(|x|+|y|)}[[z, x], y]=0 .}
\end{gathered}
$$

In particular, $\mathfrak{g}_{\overline{0}}$ is a Lie algebra and $\mathfrak{g}_{\overline{1}}$ is a $\mathfrak{g}_{\overline{0}}$-module.

A simple Lie superalgebra $\mathfrak{g}$ is of classical type if the representation of $\mathfrak{g}_{\overline{0}}$ on $\mathfrak{g}_{\overline{1}}$ is totally reducible, other wise it is of the Cartan type. A simple Lie superalgebra $\mathfrak{g}$ of classical type is of type $I$ if the representation of $\mathfrak{g}_{\overline{0}}$ in $\mathfrak{g}_{\overline{1}}$ is irreducible and it is of type $I I$ if $\mathfrak{g}_{\overline{1}}$ is a direct sum of two irreducible $\mathfrak{g}_{0}$-modules.

Consider several examples.

Consider the vector superspace $V=V_{\overline{0}} \oplus V_{\overline{1}}$. The Lie algebra of all endomorphisms of $V$ is denoted by $\mathfrak{g l}(V)$. Its even part $\mathfrak{g l}(V)_{\overline{0}}=\mathfrak{g l}\left(V_{\overline{0}}\right) \oplus \mathfrak{g l}\left(V_{\overline{1}}\right)$ consists of even endomorphisms, i.e. 
of morphisms; the odd part of $\mathfrak{g l}(V)$ consists of odd endomorphisms, i.e. of the endomorphisms changing the parity and it holds $\mathfrak{g l}(V)_{\overline{1}}=V_{\overline{0}}^{*} \otimes V_{\overline{1}} \oplus V_{\overline{1}}^{*} \otimes V_{\overline{0}}$.

Consider the vector superspace $V=\mathbb{R}^{n \mid m}=\mathbb{R}^{n} \oplus \Pi \mathbb{R}^{m}$. Here $\Pi$ is the parity changing functor, in particular it is used to show that the vector space $\Pi \mathbb{R}^{m}$ is purely odd. In this case $\mathfrak{g l}(V)$ is denote by $\mathfrak{g l}(n \mid m, \mathbb{R})$ (if $V$ is a real vector superspace). In the matrix form we have

$$
\mathfrak{g l}(n \mid m, \mathbb{R})=\left\{\left(\begin{array}{cc}
A & B \\
C & D
\end{array}\right)\right\}
$$

where

$$
\mathfrak{g l}(n \mid m, \mathbb{R})_{\overline{0}}=\left\{\left(\begin{array}{cc}
A & 0 \\
0 & D
\end{array}\right)\right\}, \quad \mathfrak{g l}(n \mid m, \mathbb{R})_{\overline{1}}=\left\{\left(\begin{array}{cc}
0 & B \\
C & 0
\end{array}\right)\right\} .
$$

Consider the Lie superalgebra $\mathfrak{s l}(n \mid m, \mathbb{R})=\{\xi \in \mathfrak{g l}(n \mid m, \mathbb{R}) \mid \operatorname{str} \xi=0\}$, where

$$
\operatorname{str}\left(\begin{array}{cc}
A & B \\
C & D
\end{array}\right)=\operatorname{tr} A-\operatorname{tr} D .
$$

If $n \neq m$, then the Lie superalgebra $\mathfrak{s l}(n \mid m)$ is simple of the classical type II.

Let $g$ be an even non-degenerate supersymmetric form on the vector superspace $\mathbb{R}^{n \mid 2 m}=\mathbb{R}^{n} \oplus$ $\Pi \mathbb{R}^{2 m}$, i.e. $g\left(\mathbb{R}^{n}, \Pi \mathbb{R}^{2 m}\right)=g\left(\Pi \mathbb{R}^{2 m}, \mathbb{R}^{n}\right)=0$, the restriction $\eta$ of $g$ to $\mathbb{R}^{n}$ is non-degenerate and symmetric (with some signature $(p, q), p+q=n$ ), and the restriction $\omega$ of $g$ to $\mathbb{R}^{2 m}$ is non-degenerate and skew-symmetric. Having such form, we denote $\mathbb{R}^{n \mid 2 m}$ also by $\mathbb{R}^{p, q \mid 2 m}$. The orthosymplectic Lie superalgebra is defined as the supersubalgebra of $\mathfrak{g l}(n \mid 2 m, \mathbb{R})$ preserving $g$,

$$
\mathfrak{o s p}(p, q \mid 2 m)_{\bar{i}}=\left\{\xi \in \mathfrak{g l}(n \mid 2 m, \mathbb{R})_{\bar{i}} \mid g(\xi x, y)+(-1)^{|x| \bar{i}} g(x, \xi y)=0\right\}, \bar{i} \in \mathbb{Z}_{2} .
$$

If $p+q \neq 2$, then the Lie superalgebra $\mathfrak{o s p}(p, q \mid 2 m)$ is simple of classical type I. In particular, if the restriction of $g$ to $\mathbb{R}^{n}$ is positive definite, choose a basis with respect to which the matrix of $g$ has the form $\left(\begin{array}{ccc}1_{n} & 0 & 0 \\ 0 & 0 & 1_{m} \\ 0 & -1_{m} & 0\end{array}\right)$. Then,

$$
\mathfrak{o s p}(n \mid 2 m)=\left\{\left(\begin{array}{ccc}
A & B_{1} & B_{2} \\
B_{2}^{t} & C_{1} & C_{2} \\
-B_{1}^{t} & C_{3} & -C_{1}^{t}
\end{array}\right) \mid A^{t}=-A, C_{2}^{t}=C_{2}, C_{3}^{t}=C_{3}\right\} .
$$

Similarly, the Lie superalgebra $\mathfrak{o s p}^{s k}(2 k \mid p, q), p+q=m$, is defined as the supersubalgebra of $\mathfrak{g l}(2 k \mid m, \mathbb{R})$ preserving an even non-degenerate supersymmetric form on the vector superspace $\mathbb{R}^{2 k} \oplus \Pi \mathbb{R}^{m}$, in this case the restriction of $g$ to $\mathbb{R}^{2 k}$ is non-degenerate and skew-symmetric, and the restriction of $g$ to $\mathbb{R}^{m}$ is non-degenerate and symmetric (with the signature $(p, q), p+q=m$ ). For example,

$$
\mathfrak{o s p}(2 k \mid m)=\left\{\left(\begin{array}{ccc}
C_{1} & C_{2} & B_{1} \\
C_{3} & -C_{1}^{t} & B_{2} \\
B_{2}^{t} & -B_{1}^{t} & A
\end{array}\right) \mid A^{t}=-A, C_{2}^{t}=C_{2}, C_{3}^{t}=C_{3}\right\}
$$

Suppose that $n$ is even and suppose that we have an even non-degenerate supersymmetric form on the vector superspace $\mathbb{R}^{n} \oplus \Pi \mathbb{R}^{2 m}$ such that the restriction of $g$ to $\mathbb{R}^{n}$ has signature $\left(2 p_{0}, 2 q_{0}\right), 2 p_{0}+2 q_{0}=n$. Suppose that we have also a complex structure $J$ on $\mathbb{R}^{n} \oplus \Pi \mathbb{R}^{2 m}$ such that $g(J x, J y)=g(x, y)$ for all $x, y \in \mathbb{R}^{n} \oplus \Pi \mathbb{R}^{2 m}$. Note that the restriction of $g(J \cdot, \cdot)$ to $\mathbb{R}^{2 m}$ is symmetric and non-degenerate and let this form have the signature $\left(2 p_{1}, 2 q_{1}\right), p_{1}+q_{1}=m$. By definition,

$$
\mathfrak{u}\left(p_{0}, q_{0} \mid p_{1}, q_{1}\right)=\left\{\xi \in \mathfrak{o s p}\left(2 p_{0}, 2 q_{0} \mid 2 m\right) \mid[\xi, J]=0\right\}
$$




$$
\mathfrak{s u}\left(p_{0}, q_{0} \mid p_{1}, q_{1}\right)=\left\{\xi \in \mathfrak{u}\left(p_{0}, q_{0} \mid p_{1}, q_{1}\right) \mid \operatorname{str}(J \circ \xi)=0\right\} .
$$

Similarly, suppose that $n$ and $2 m$ are integers divided by $4, m=2 k$, and suppose that we have an even non-degenerate supersymmetric form on the vector superspace $\mathbb{R}^{n} \oplus \Pi \mathbb{R}^{2 m}$ such that the restriction of $g$ to $\mathbb{R}^{n}$ has the signature $(4 r, 4 s), 4 r+4 s=n$. Suppose that we have a quaternionic structure $I, J, K$ on $\mathbb{R}^{n} \oplus \Pi \mathbb{R}^{2 m}$ (i.e. $I, J, K$ are complex structures and they generate the Lie algebra isomorphic to $\mathfrak{s p}(1))$ such that $g(I x, I y)=g(J x, J y)=g(K x, K y)=$ $g(x, y)$ for all $x, y \in \mathbb{R}^{n} \oplus \Pi \mathbb{R}^{2 m}$. By definition,

$$
\mathfrak{h o s p}(r, s \mid k)=\{\xi \in \mathfrak{o s p}(4 r, 4 s \mid 2 m) \mid[\xi, I]=[\xi, J]=[\xi, K]=0\} .
$$

Note that the normalizer of $\mathfrak{s p}(1)$ in $\mathfrak{o} \mathfrak{s p}(4 r, 4 s \mid 2 m)$ coincides with $\mathfrak{s p}(1) \oplus \mathfrak{h} \mathfrak{o s p}(r, s \mid k)$. Remark also that $\mathfrak{h o s p}(r, s \mid k)_{\overline{0}} \cap \mathfrak{s p}(2 m, \mathbb{R})=\mathfrak{s o}^{*}(k)$, where $\mathfrak{s o}^{*}(k)$ is the subalgebra of $\mathfrak{g l}(k, \mathbb{H})$ acting on $\mathbb{R}^{4 k}=\mathbb{H}^{k}$ and preserving the skew-quaternionic-Hermitian form

$$
\omega(\cdot, \cdot)+i \omega(I \cdot, \cdot)+j \omega(J \cdot, \cdot)+k \omega(K \cdot, \cdot),
$$

here $\omega$ is the restriction of $g$ to $\mathbb{R}^{4 k}$ considered as a skew-symmetric bilinear form on $\mathbb{R}^{4 k}$.

\section{Supermanifolds and their holonomy groups}

The introduction to the theory of supermanifolds can be found in [11, 26, 28, 39. Riemannian supermanifolds are considered e.g. in [3, 17, 9, 10, 18]. The holonomy theory is introduced in [15].

A real smooth supermanifold $\mathcal{M}$ of dimension $n \mid m$ is a pair $\left(M, \mathcal{O}_{\mathcal{M}}\right)$, where $M$ is a Hausdorff topological space and $\mathcal{O}_{\mathcal{M}}$ is a sheaf of commutative superalgebras with unity over $\mathbb{R}$ locally isomorphic to

$$
\mathbb{R}^{n ; m}=\left(\mathbb{R}^{n}, \mathcal{O}_{\mathbb{R}^{n ; m}}=\mathcal{O}_{\mathbb{R}^{n}} \otimes \Lambda(m)\right),
$$

where $\mathcal{O}_{\mathbb{R}^{n}}$ is the sheaf of smooth functions on $\mathbb{R}^{n}$ and $\Lambda(m)$ is the Grassmann superalgebra of $m$ generators. The sections of the sheaf $\mathcal{O}_{\mathcal{M}}$ are called superfunctions (or just functions) on $\mathcal{M}$. The ideal $\left(\mathcal{O}_{\mathcal{M}}\right)_{\overline{1}} \oplus\left(\left(\mathcal{O}_{\mathcal{M}}\right)_{\overline{1}}\right)^{2}$ consists of the nilpotent elements of $\mathcal{O}_{\mathcal{M}}$, and the sheaf $\mathcal{O}_{M}$ defined as the quotient $\mathcal{O}_{\mathcal{M}} /\left(\left(\mathcal{O}_{\mathcal{M}}\right)_{\overline{1}} \oplus\left(\left(\mathcal{O}_{\mathcal{M}}\right)_{\overline{1}}\right)^{2}\right)$ furnish $M$ with the structure of a real smooth manifold. We get the canonical projection $\sim: \mathcal{O}_{\mathcal{M}} \rightarrow \mathcal{O}_{M}, f \mapsto \tilde{f}$. The value of a superfunction $f$ at a point $x \in M$ is by definition $\tilde{f}(x)$. If $m=0$, then $\mathcal{M}=M$ is a smooth manifold.

Denote by $\mathcal{T}_{\mathcal{M}}$ the tangent sheaf, i.e. the sheaf of superderivatives of the sheaf $\mathcal{O}_{\mathcal{M}}$. For a point $x \in M$ define the tangent space

$$
T_{x} \mathcal{M}=\mathcal{T}_{\mathcal{M}}(U) /\left(\mathcal{O}_{\mathcal{M}}(U)_{x} \mathcal{T}_{\mathcal{M}}(U)\right),
$$

where $U \subset M$ is any open subset containing $x$ and $\mathcal{O}_{\mathcal{M}}(U)_{x}$ is the ideal in $\mathcal{O}_{\mathcal{M}}(U)$ consisting of functions vanishing at the point $x$. The space $T_{x} \mathcal{M}$ is a vector superspace of the same dimension as $\mathcal{M}$ and its even part coincides with $T_{x} M$. Consider the tangent bundle $T \mathcal{M}=\cup_{x \in M} T_{x} \mathcal{M}$ as a bundle of vector superspaces over $M$, its even part is the tangent bundle of $M$. We may consider also $T \mathcal{M}$ as a usual vector bundle over $M$. For a section $X \in \mathcal{T}_{\mathcal{M}}(U)$, its value $X_{x} \in T_{x} \mathcal{M}$ is defined in the obvious way. We get the natural projection $\sim: \mathcal{T}_{\mathcal{M}}(U) \rightarrow \Gamma(T \mathcal{M}, U)$.

$A$ connection on $\mathcal{M}$ is an even morphism $\nabla: \mathcal{T}_{\mathcal{M}} \otimes_{\mathbb{R}} \mathcal{T}_{\mathcal{M}} \rightarrow \mathcal{T}_{\mathcal{M}}$ of sheaves of supermodules over $\mathbb{R}$ such that

$$
\nabla_{f Y} X=f \nabla_{Y} X \quad \text { and } \quad \nabla_{Y} f X=(Y f) X+(-1)^{|Y||f|} f \nabla_{Y} X
$$

for all homogeneous functions $f$, vector fields $X, Y$ on $\mathcal{M}$, here $|\cdot| \in \mathbb{Z}_{2}=\{\overline{0}, \overline{1}\}$ denotes the parity. In particular, $\left|\nabla_{Y} X\right|=|Y|+|X|$. The curvature tensor of the connection $\nabla$ is given by

$$
R(Y, Z)=\left[\nabla_{Y}, \nabla_{Z}\right]-\nabla_{[Y, Z]},
$$


where $Y$ and $Z$ are vector fields on $\mathcal{M}$.

Obviously, the restriction

$$
\tilde{\nabla}=\left(\left.\nabla\right|_{\Gamma(T M) \otimes \Gamma(T \mathcal{M})}\right)^{\sim}: \Gamma(T M) \otimes \Gamma(T \mathcal{M}) \rightarrow \Gamma(T \mathcal{M})
$$

is a connection on the vector bundle $T \mathcal{M}$. Since $\nabla$ is even, the subbundles $T M=(T \mathcal{M})_{\overline{0}},(T \mathcal{M})_{\overline{1}} \subset$ $T \mathcal{M}$ are parallel. We obtain also a linear connection on $M$. Let $\gamma:[a, b] \subset \mathbb{R} \rightarrow M$ be a curve and $\tau_{\gamma}: T_{\gamma(a)} \mathcal{M} \rightarrow T_{\gamma(b)} \mathcal{M}$ the parallel displacement along $\gamma$ defined by the connection $\tilde{\nabla}$.

The holonomy algebra $\mathfrak{h o l}(\nabla)_{x}$ of the connection $\nabla$ at a point $x \in M$ is the supersubalgebra of the Lie superalgebra $\mathfrak{g l}\left(T_{x} \mathcal{M}\right)$ generated by the operators of the form

$$
\tau_{\gamma}^{-1} \circ \nabla_{Y_{r}, \ldots, Y_{1}}^{r} R_{y}(Y, Z) \circ \tau_{\gamma}: T_{x} \mathcal{M} \rightarrow T_{x} \mathcal{M}
$$

where $\gamma$ is any piecewise smooth curve in $M$ beginning at the point $x ; y \in M$ is the end-point of the curve $\gamma, r \geq 0$, and $Y, Z, Y_{1}, \ldots, Y_{r} \in T_{y} \mathcal{M}$.

Now we define the holonomy group. Recall that a Lie supergroup $\mathcal{G}=\left(G, \mathcal{O}_{\mathcal{G}}\right)$ is a group object in the category of supermanifolds. The underlying smooth manifold $G$ is a Lie group. The Lie superalgebra $\mathfrak{g}$ of $\mathcal{G}$ can be identified with the tangent space to $\mathcal{G}$ at the identity $e \in G$. The Lie algebra of the Lie group $G$ is the even part $\mathfrak{g}_{\overline{0}}$ of the Lie superalgebra $\mathfrak{g}$. Any Lie supergroup $\mathcal{G}$ is uniquely given by a pair $(G, \mathfrak{g})$ (Harish-Chandra pair), where $G$ is a Lie group, $\mathfrak{g}=\mathfrak{g}_{\overline{0}} \oplus \mathfrak{g}_{\overline{1}}$ is a Lie superalgebra such that $\mathfrak{g}_{\overline{0}}$ is the Lie algebra of the Lie group $G$ and a certain conditions hold [11, 18].

Denote by $\operatorname{Hol}(\nabla)_{x}^{0}$ the connected Lie subgroup of the Lie group $\operatorname{GL}\left(\left(T_{x} \mathcal{M}\right)_{\overline{0}}\right) \times \operatorname{GL}\left(\left(T_{x} \mathcal{M}\right)_{\overline{1}}\right)$ corresponding to the Lie subalgebra $\left(\mathfrak{h o l}(\nabla)_{x}\right)_{\overline{0}} \subset \mathfrak{g l}\left(\left(T_{x} \mathcal{M}\right)_{\overline{0}}\right) \oplus \mathfrak{g l}\left(\left(T_{x} \mathcal{M}\right)_{\overline{1}}\right) \subset \mathfrak{g l}\left(T_{x} \mathcal{M}\right)$. Let $\operatorname{Hol}(\nabla)_{x}$ be the Lie subgroup of the Lie group $\operatorname{GL}\left(\left(T_{x} \mathcal{M}\right)_{\overline{0}}\right) \times \operatorname{GL}\left(\left(T_{x} \mathcal{M}\right)_{\overline{1}}\right)$ generated by the Lie groups $\operatorname{Hol}(\nabla)_{x}^{0}$ and $\operatorname{Hol}(\tilde{\nabla})_{x}$. Clearly, the Lie algebra of the Lie group $\operatorname{Hol}(\nabla)_{x}$ is $\left(\mathfrak{h o r}(\nabla)_{x}\right)_{\overline{0}}$. The Lie supergroup $\mathcal{H o l}(\nabla)_{x}$ given by the Harish-Chandra pair $\left(\operatorname{Hol}(\nabla)_{x}, \mathfrak{h o l}(\nabla)_{x}\right)$ is called the holonomy group of the connection $\nabla$ at the point $x$. The Lie supergroup $\mathcal{H}$ ol $(\nabla)_{x}^{0}$ given by the Harish-Chandra pair $\left(\operatorname{Hol}(\nabla)_{x}^{0}, \mathfrak{h o l}(\nabla)_{x}\right)$ is called the restricted holonomy group of the connection $\nabla$ at the point $x$.

If $\mathcal{M}=M$ is a usual smooth manifold and $\nabla$ is a connection on $M$, than $\mathcal{H o l}(\nabla)_{x}$ and $\mathfrak{h o r}(\nabla)_{x}$ coincide with the holonomy group and the holonomy algebra of $(M, \nabla)$, respectively.

If the manifold $M$ is simply connected, then the group $\operatorname{Hol}(\nabla)_{x}$ is connected and the whole information about $\mathcal{H o l}(\nabla)_{x}$ gives us the holonomy algebra $\mathfrak{h o l}(\nabla)_{x}$.

A Riemannian supermanifold $(\mathcal{M}, g)$ is a supermanifold $\mathcal{M}$ of dimension $n \mid 2 m$ endowed with an even non-degenerate supersymmetric metric

$$
g: \mathcal{T}_{\mathcal{M}} \otimes_{\mathcal{O}_{\mathcal{M}}} \mathcal{T}_{\mathcal{M}} \rightarrow \mathcal{O}_{\mathcal{M}}
$$

see e.g. [10]. In particular, the value $g_{x}$ of $g$ at a point $x \in M$ satisfies: $g_{x}\left(\left(T_{x} \mathcal{M}\right)_{\overline{0}},\left(T_{x} \mathcal{M}\right)_{\overline{1}}\right)=$ $0,\left.g_{x}\right|_{\left(T_{x} \mathcal{M}\right)_{\overline{0}} \times\left(T_{x} \mathcal{M}\right)_{\overline{0}}}$ is non-degenerate, symmetric and $\left.g_{x}\right|_{\left(T_{x} \mathcal{M}\right)_{\overline{1}} \times\left(T_{x} \mathcal{M}\right)_{\overline{1}}}$ is non-degenerate, skewsymmetric. The metric $g$ defines a pseudo-Riemannian metric $\tilde{g}$ on the manifold $M$. Note that $\tilde{g}$ is not assumed to be positively defined. The supermanifold $(\mathcal{M}, g)$ has a unique linear connection $\nabla$ such that $\nabla$ is torsion-free and $\nabla g=0$. This connection is called the LeviCivita connection. We denote the holonomy algebra of the connection $\nabla$ by $\mathfrak{h o l}(\mathcal{M}, g)$. Since $g$ is parallel, $\mathfrak{h o l}(\mathcal{M}, g) \subset \mathfrak{o s p}(p, q \mid 2 m)$ and $\operatorname{Hol}(\tilde{\nabla}) \subset \mathrm{O}(p, q) \times \operatorname{Sp}(2 m, \mathbb{R})$, where $(p, q)$ is the signature of the pseudo-Riemannian metric $\tilde{g}$.

We call a supersubalgebra $\mathfrak{g} \subset \mathfrak{o s p}(p, q \mid 2 m)$ weakly-irreducible if it does not preserve any nondegenerate vector supersubspace of $\mathbb{R}^{p, q} \oplus \Pi \mathbb{R}^{2 m}$. The following theorem generalizes the $\mathrm{Wu}$ theorem [40]. 
Theorem 1 [15] Let $(\mathcal{M}, g)$ be a Riemannian supermanifold such that the pseudo-Riemannian manifold $(M, \tilde{g})$ is simply connected and geodesically complete. Then there exist Riemannian supermanifolds $\left(\mathcal{M}_{0}, g_{0}\right),\left(\mathcal{M}_{1}, g_{1}\right), \ldots,\left(\mathcal{M}_{r}, g_{r}\right)$ such that

$$
(\mathcal{M}, g)=\left(\mathcal{M}_{0} \times \mathcal{M}_{1} \times \cdots \times \mathcal{M}_{r}, g_{0}+g_{1}+\cdots+g_{r}\right)
$$

the supermanifold $\left(\mathcal{M}_{0}, g_{0}\right)$ is flat and the holonomy algebras of the supermanifolds $\left(\mathcal{M}_{1}, g_{1}\right), \ldots,\left(\mathcal{M}_{r}, g_{r}\right)$ are weakly-irreducible. In particular,

$$
\mathfrak{h o l}(\mathcal{M}, g)=\mathfrak{h o l}\left(\mathcal{M}_{1}, g_{1}\right) \oplus \cdots \oplus \mathfrak{h o l}\left(\mathcal{M}_{r}, g_{r}\right)
$$

For general $(\mathcal{M}, g)$ decomposition (3) holds locally.

\section{$5 \quad$ Berger superalgebras}

Let $V=V_{\overline{0}} \oplus V_{\overline{1}}$ be a real or complex vector superspace and $\mathfrak{g} \subset \mathfrak{g l}(V)$ a supersubalgebra. The space of algebraic curvature tensors of type $\mathfrak{g}$ is the vector superspace $\mathcal{R}(\mathfrak{g})=\mathcal{R}(\mathfrak{g})_{\overline{0}} \oplus \mathcal{R}(\mathfrak{g})_{\overline{1}}$, where

$$
\mathcal{R}(\mathfrak{g})=\left\{\begin{array}{l|l}
R \in \Lambda^{2} V^{*} \otimes \mathfrak{g} & \begin{array}{c}
R(X, Y) Z+(-1)^{|X|(|Y|+|Z|)} R(Y, Z) X \\
+(-1)^{|Z|(|X|+|Y|)} R(Z, X) Y=0 \\
\text { for all homogeneous } X, Y, Z \in V
\end{array}
\end{array}\right\} .
$$

Here $|\cdot| \in \mathbb{Z}_{2}$ denotes the parity. The identity that satisfy the elements $R \in \mathcal{R}(\mathfrak{g})$ is called the first Bianchi super identity. Obviously, $\mathcal{R}(\mathfrak{g})$ is a $\mathfrak{g}$-module with respect to the action $A \cdot R=R_{A}$,

$$
R_{A}(X, Y)=[A, R(X, Y)]-(-1)^{|A||R|} R(A X, Y)-(-1)^{|A|(|R|+|X|)} R(X, A Y),
$$

where $A \in \mathfrak{g}, R \in \mathcal{R}(\mathfrak{g})$ and $X, Y \in V$ are homogeneous.

If $\mathcal{M}$ is a supermanifold and $\nabla$ is a linear torsion-free connection on the tangent sheaf $\mathcal{T}_{\mathcal{M}}$ with the holonomy algebra $\mathfrak{h o l}(\nabla)_{x}$ at some point $x$, then for the covariant derivatives of the curvature tensor we have $\left(\nabla_{Y_{r}, \ldots, Y_{1}}^{r} R\right)_{x} \in \mathcal{R}\left(\mathfrak{h o l}(\nabla)_{x}\right)$ for all $r \geq 0$ and tangent vectors $Y_{1}, \ldots, Y_{r} \in T_{x} M$. Moreover, $\left|\left(\nabla_{Y_{r}, \ldots, Y_{1}}^{r} R\right)_{x}\right|=\left|Y_{1}\right|+\cdots+\left|Y_{r}\right|$, whenever $Y_{1}, \ldots, Y_{r}$ are homogeneous.

Define the vector supersubspace

$$
L(\mathcal{R}(\mathfrak{g}))=\operatorname{span}\{R(X, Y) \mid R \in \mathcal{R}(\mathfrak{g}), X, Y \in V\} \subset \mathfrak{g} .
$$

From (5) it follows that $L(\mathcal{R}(\mathfrak{g}))$ is an ideal in $\mathfrak{g}$. We call a supersubalgebra $\mathfrak{g} \subset \mathfrak{g l}(V)$ a Berger superalgebra if $L(\mathcal{R}(\mathfrak{g}))=\mathfrak{g}$.

If $V$ is a vector space, which can be considered as a vector superspace with the trivial odd part, then $\mathfrak{g} \subset \mathfrak{g l}(V)$ is a usual Lie algebra, which can be considered as a Lie superalgebra with the trivial odd part. Berger superalgebras in this case are the same as the usual Berger algebras.

Proposition 1 [15] Let $\mathcal{M}$ be a supermanifold of dimension $n \mid m$ with a linear torsion-free connection $\nabla$. Then its holonomy algebra $\mathfrak{h o l}(\nabla) \subset \mathfrak{g l}(n \mid m, \mathbb{R})$ is a Berger superalgebra.

Consider the vector superspace

$$
\mathcal{R}^{\nabla}(\mathfrak{g})=\left\{\begin{array}{l|l}
S \in V^{*} \otimes \mathcal{R}(g) & \begin{array}{c}
S_{X}(Y, Z)+(-1)^{|X|(|Y|+|Z|)} S_{Y}(Z, X) \\
+(-1)^{|Z|(|X|+|Y|)} S_{Z}(X, Y)=0 \\
\text { for all homogeneous } X, Y, Z \in V
\end{array}
\end{array}\right\} .
$$


If $\mathcal{M}$ is a supermanifold and $\nabla$ is a linear torsion-free connection on $\mathcal{T}_{\mathcal{M}}$, then $\left(\nabla_{Y_{r}, \ldots, Y_{2},}^{r} R\right)_{x} \in$ $\mathcal{R}^{\nabla}\left(\mathfrak{h o l}(\nabla)_{x}\right)$ for all $r \geq 1$ and $Y_{2}, \ldots, Y_{r} \in T_{x} M$. Moreover, $\left|\left(\nabla_{Y_{r}, \ldots, Y_{2},}^{r} R\right)_{x}\right|=\left|Y_{2}\right|+\cdots+\left|Y_{r}\right|$, whenever $Y_{2}, \ldots, Y_{r}$ are homogeneous.

A Berger superalgebra $\mathfrak{g}$ is called symmetric if $\mathcal{R}^{\nabla}(\mathfrak{g})=0$. This is a generalization of the usual symmetric Berger algebras, see e.g. [34, and the following is a generalization of the well-known fact about smooth manifolds.

Proposition 2 [15] Let $\mathcal{M}$ be a supermanifold with a torsion free connection $\nabla$. If $\mathfrak{h o l}(\nabla)$ is a symmetric Berger superalgebra, then $(\mathcal{M}, \nabla)$ is locally symmetric (i.e. $\nabla R=0)$. If $(\mathcal{M}, \nabla)$ is a locally symmetric superspace, then its curvature tensor at any point is annihilated by the holonomy algebra at this point and its image coincides with the holonomy algebra.

A geometric theory of Riemannian symmetric superspaces is developed recently in [18].

The proof of the following proposition is as in [34].

Proposition 3 Let $\mathfrak{g} \subset \mathfrak{g l}(V)$ be an irreducible Berger superalgebra of the form (1). If $\mathfrak{g}$ annihilates the module $\mathcal{R}(\mathfrak{g})$, then $\mathfrak{g}$ is a symmetric Berger superalgebra.

In [38] simply connected symmetric superspaces of simple Lie supergroups of isometries are classified. In particular this implies the classification of the holonomy algebras of Riemannian symmetric superspaces and of irreducible Berger superalgebras $\mathfrak{g} \subset \mathfrak{o s p}(p, q \mid 2 m)$ of the form (11). Hence we assume that the Riemannian supermanifolds under the consideration are not locally symmetric.

\section{The Main Results}

Here is the Main Theorem of this paper.

Theorem 2 Let $(\mathcal{M}, g)$ be a not locally symmetric Riemannian supermanifold of dimension $p+q \mid 2 m(p+q>0)$ with an irreducible holonomy algebra $\mathfrak{h o l}(\mathcal{M}, g) \subset \mathfrak{o s p}(p, q \mid 2 m)$ that is of the form (1), then $\mathfrak{h o l}(\mathcal{M}, g) \subset \mathfrak{o s p}(p, q \mid 2 m)$ coincides with one of the Lie superalgebras from Table 1 .

Table 1 Irreducible non-symmetric Berger supersubalgebras $\mathfrak{g} \subset \mathfrak{o s p}(p, q \mid 2 m)(p+q>0)$ of the from (11) and the connected Lie subgroups $G \subset \mathrm{SO}(p, q) \times \mathrm{Sp}(2 m, \mathbb{R})$ corresponding to $\mathfrak{g}_{\overline{0}} \subset \mathfrak{s o}(p, q) \oplus \mathfrak{s p}(2 m, \mathbb{R})$.

\begin{tabular}{|c|c|c|}
\hline $\mathfrak{g}$ & $\bar{G}$ & $(p, q \mid 2 m)$ \\
\hline $\mathfrak{o} \mathfrak{s p}(p, q \mid 2 m)$ & $\mathrm{SO}(p, q) \times \mathrm{Sp}(2 m, \mathbb{R})$ & $(p, q \mid 2 m)$ \\
\hline $\mathfrak{o s p}(p \mid 2 k, \mathbb{C})$ & $\mathrm{SO}(p, \mathbb{C}) \times \mathrm{Sp}(2 k, \mathbb{R})$ & $(p, p \mid 4 k)$ \\
\hline $\mathfrak{u}\left(p_{0}, q_{0} \mid p_{1}, q_{1}\right)$ & $\mathrm{U}\left(p_{0}, q_{0}\right) \times \mathrm{U}\left(p_{1}, q_{1}\right)$ & $\left(2 p_{0}, 2 q_{0} \mid 2 p_{1}+2 q_{1}\right)$ \\
\hline $\mathfrak{s u}\left(p_{0}, q_{0} \mid p_{1}, q_{1}\right)$ & $\mathrm{U}(1)\left(\mathrm{SU}\left(p_{0}, q_{0}\right) \times \mathrm{SU}\left(p_{1}, q_{1}\right)\right)$ & $\left(2 p_{0}, 2 q_{0} \mid 2 p_{1}+2 q_{1}\right)$ \\
\hline $\mathfrak{h o s p}(r, s \mid k)$ & $\operatorname{Sp}\left(p_{0}, q_{0}\right) \times \mathrm{SO}^{*}(k)$ & $(4 r, 4 s \mid 4 k)$ \\
\hline $\mathfrak{h o s p}(r, s \mid k) \oplus \mathfrak{s p}(1)$ & $\operatorname{Sp}(1)\left(\operatorname{Sp}\left(p_{0}, q_{0}\right) \times \mathrm{SO}^{*}(k)\right)$ & $(4 r, 4 s \mid 4 k)$ \\
\hline $\mathfrak{o} \mathfrak{s p}^{s k}(2 k \mid r, s) \oplus \mathfrak{s l}(2, \mathbb{R})$ & $\operatorname{Sp}(2 k, \mathbb{R}) \times \operatorname{SO}(r, s) \times \operatorname{SL}(2, \mathbb{R})$ & $(2 k, 2 k \mid 2 r+2 s)$ \\
\hline $\mathfrak{o s p}^{s k}(2 k \mid r, \mathbb{C}) \oplus \mathfrak{s l}(2, \mathbb{C})$ & $\operatorname{Sp}(2 k, \mathbb{C}) \times \operatorname{SO}(r, \mathbb{C}) \times \operatorname{SL}(2, \mathbb{C})$ & $(4 k, 4 k \mid 4 r)$ \\
\hline
\end{tabular}


The Ricci tensor of a supermanifold $(\mathcal{M}, g)$ is defined by the formula

$$
\operatorname{Ric}(Y, Z)=\operatorname{str}\left(X \mapsto(-1)^{|X||Z|} R(Y, X) Z\right),
$$

where $U \subset M$ is open and $X, Y, Z \in \mathcal{T}_{\mathcal{M}}(U)$ are homogeneous.

The definitions of the supermanifolds considered below are similar to the usual ones, see e.g. [10, 15. Foe example, a Riemannian supermanifold $(\mathcal{M}, g)$ is called a Kählerian supermanifold if it admits an even parallel $g$-orthogonal complex structure. Since the holonomy algebra annihilates the values of the parallel tensors [15], in this case it must be contained in $\mathfrak{u}\left(p_{0}, q_{0} \mid p_{1}, q_{1}\right)$. By definition, a special Kählerian supermanifold or a Calabi-Yau supermanifold is a Ricci-flat Kählerian supermanifold.

Proposition 4 [15] Let $(\mathcal{M}, g)$ be a Kählerian supermanifold, then $\mathrm{Ric}=0$ if and only if $\mathfrak{h o l}(\mathcal{M}, g) \subset \mathfrak{s u}\left(p_{0}, q_{0} \mid p_{1}, q_{1}\right)$.

Riemannian supermanifolds with the holonomy algebras $\mathfrak{o s p}(p, q \mid 2 m)$ are generic. Here we give the geometric characterization of simply connected supermanifolds with the holonomy algebras $\mathfrak{g}$ different from $\mathfrak{o s p}(p, q \mid 2 m)$ (the conditions on the holonomy algebra and the corresponding geometries are equivalent for simply connected supermanifolds):

$\mathfrak{g} \subset \mathfrak{o s p}(p \mid 2 k, \mathbb{C}):$ holomorphic Riemannian supermanifolds;

$\mathfrak{g} \subset \mathfrak{u}\left(p_{0}, q_{0} \mid p_{1}, q_{1}\right):$ Kählerian supermanifolds;

$\mathfrak{g} \subset \mathfrak{s u}\left(p_{0}, q_{0} \mid p_{1}, q_{1}\right):$ special Kählerian supermanifolds or Calabi-Yau supermanifolds;

$\mathfrak{g} \subset \mathfrak{h o s p}(r, s \mid k)$ : hyper-Kählerian supermanifolds;

$\mathfrak{g} \subset \mathfrak{h o s p}(r, s \mid k) \oplus \mathfrak{s p}(1):$ quaternionic-Kählerian supermanifolds;

$\mathfrak{g} \subset \mathfrak{o s p}^{s k}(2 k \mid r, s) \oplus \mathfrak{s l}(2, \mathbb{R}):$ para-Kählerian supermanifolds;

$\mathfrak{g} \subset \mathfrak{o s p}^{s k}(2 k \mid r, \mathbb{C}) \oplus \mathfrak{s l}(2, \mathbb{C}):$ holomorphic para-Kählerian supermanifolds.

Proposition 5 [15] Let $(\mathcal{M}, g)$ be a quaternionic-Kählerian supermanifold, then Ric $=0$ if and only if $\mathfrak{h o l}(\mathcal{M}, g) \subset \mathfrak{h o s p}\left(p_{0}, q_{0} \mid p_{1}, q_{1}\right)$. In particular, if $(\mathcal{M}, g)$ is hyper-Kählerian, then Ric $=0 ;$ if $M$ is simply connected, $(\mathcal{M}, g)$ is quaternionic-Kählerian and $\operatorname{Ric}=0$, then $(\mathcal{M}, g)$ is hyper-Kählerian.

Now the natural problem is to construct examples of supermanifolds with each of the obtained possible holonomy algebras. Note that examples of special Kählerian manifolds (i.e. CalabiYau manifolds) delivers the Calabi-Yau Theorem. In [33] it is shown that the Calabi-Yau Theorem does not hold for Kählerian supermanifolds of real odd dimension two. In [41] it is shown that the arguments of [33] work only for the odd dimension two and it is conjectured that Calabi-Yau Theorem is true for manifolds of odd dimensions bigger then two. In [1] some examples of Calabi-Yau supermanifolds are constructed. Examples of quaternionic-Kählerian supermanifolds are constructed in [10].

The rest sections are dedicated to the proof of Theorem 2 . 


\section{$7 \quad$ Weak-Berger algebras}

Let $\mathfrak{g} \subset \mathfrak{s o}(p, q)=\mathfrak{s o}(V)$ be a subalgebra. Denote by $\eta$ the pseudo-Euclidian metric on $V=\mathbb{R}^{p, q}$. The vector space

$$
\mathcal{P}_{\eta}(\mathfrak{g})=\left\{\begin{array}{c|c}
P \in V^{*} \otimes \mathfrak{g} & \eta(P(X) Y, Z)+\eta(P(Y) Z, X)+\eta(P(Z) X, Y)=0 \\
\text { for all } X, Y, Z \in V
\end{array}\right\}
$$

is called the space of weak-curvature tensors of type $\mathfrak{g}$. A subalgebra $\mathfrak{g} \subset \mathfrak{s o}(V)$ is called a weak-Berger algebra if $\mathfrak{h}$ is spanned by the images of the elements $P \in \mathcal{P}(\mathfrak{h})$. It is not hard to see that if $R \in \mathcal{R}(\mathfrak{g})$ and $x \in V$ is fixed, then $R(\cdot, x) \in \mathcal{P}_{\eta}(\mathfrak{g})$. In particular, any Berger algebra is a weak-Berger algebra. The converse statement is not obvious, it is proved recently in [25].

Theorem 3 Let $\mathfrak{g} \subset \mathfrak{s o}(p, q)$ be an irreducible weak-Berger subalgebra, then it is a Berger subalgebra.

Remark that in the origin theorem $\mathfrak{g}$ is a subalgebra of $\mathfrak{s o}(n)$. The above result immediately follows from the complexification process described in [25].

The spaces $\mathcal{P}_{\eta}(\mathfrak{g})$ for irreducible Berger subalgebras $\mathfrak{g} \subset \mathfrak{s o}(n)$ are found in [14]. This result can be easily extended to the case of subalgebras $\mathfrak{g} \subset \mathfrak{s o}(p, q)$. In particular, it is proved that if $\mathfrak{g}=\mathfrak{g}^{1} \oplus \mathfrak{g}^{2} \subset \mathfrak{s o}\left(V^{1} \otimes V^{2}\right)=\mathfrak{s o}(V)$, where $\mathfrak{g}^{1} \subset \mathfrak{g l}\left(V^{1}\right)$ and $\mathfrak{g}^{2} \subset \mathfrak{g l}\left(V^{2}\right)$ are irreducible, then

$$
\mathcal{P}_{\eta}\left(\mathfrak{g}^{1} \subset \mathfrak{s o}(V)\right)=\mathcal{P}_{\eta}\left(\mathfrak{g}^{2} \subset \mathfrak{s o}(V)\right)=0,
$$

unless the complexification of $\mathfrak{g} \subset \mathfrak{s o}(V)$ coincides with

$$
\mathfrak{s p}(2 m, \mathbb{C}) \oplus \mathfrak{s l}(2, \mathbb{C}) \subset \mathfrak{s o}(4 m, \mathbb{C}) .
$$

For example, for $\mathfrak{s p}(m) \oplus \mathfrak{s p}(1) \subset \mathfrak{s o}(4 m)$ it holds

$$
\mathcal{P}_{\eta}(\mathfrak{s p}(1) \subset \mathfrak{s o}(V))=0, \quad \mathcal{P}_{\eta}(\mathfrak{s p}(m) \subset \mathfrak{s o}(V))=(\mathfrak{s p}(2 m, \mathbb{C}) \subset \mathfrak{s l}(2 m, \mathbb{C}))^{(1)} .
$$

In [14] it is shown that if $\mathfrak{g} \subset \mathfrak{s o}(n)$ is an irreducible subalgebra and $\mathcal{P}_{\eta}(\mathfrak{g}) \neq 0$ or $\mathcal{R}(\mathfrak{g}) \neq 0$, then either $\mathfrak{g}$ is a Berger subalgebra, or $\mathfrak{g}=\mathfrak{s p}\left(\frac{n}{4}\right) \oplus \mathbb{R} J$. A similar result holds for irreducible subalgebras $\mathfrak{g} \subset \mathfrak{s o}(p, q)$.

Let $\mathfrak{g} \subset \mathfrak{g l}(L)$ be an irreducible subalgebra. Then $\mathfrak{g}$ is a weakly-irreducible subalgebra of $\mathfrak{s o}\left(L \oplus L^{*}\right)=\mathfrak{s o}(p, p)$, where $p=\operatorname{dim} L$. Let $\eta$ be the natural metric on $L \oplus L^{*}$. It is easy to see that $P \in \mathcal{P}_{\eta}(\mathfrak{g})$ if and only if

$$
\left.\operatorname{pr}_{\mathfrak{g l}(L)} \circ P\right|_{L} \in(\mathfrak{g} \subset \mathfrak{g l}(L))^{(1)},\left.\quad \operatorname{pr}_{\mathfrak{g r}\left(L^{*}\right)} \circ P\right|_{L} \in\left(\mathfrak{g} \subset \mathfrak{g l}\left(L^{*}\right)\right)^{(1)} .
$$

Thus if $\mathfrak{g} \subset \mathfrak{s o}\left(L \oplus L^{*}\right)$ is a weak-Berger subalgebra, then $(\mathfrak{g} \subset \mathfrak{g l}(L))^{(1)} \neq\{0\}$ and all $\mathfrak{g} \subset \mathfrak{g l}(L)$ are known [8].

\section{The case of Riemannian odd supermanifolds}

Let $(\mathcal{M}, g)$ be a Riemannian supermanifold of dimension $0 \mid 2 m$, such supermanifolds are called odd. In this case for the holonomy algebra we get

$$
\mathfrak{g} \subset \mathfrak{o s p}(0 \mid 2 m) \simeq \mathfrak{s p}(2 m, \mathbb{R})
$$


i.e. $\mathfrak{g}$ is a usual Lie algebra acting in a purely odd vector superspace. The possible irreducible holonomy algebras of such supermanifolds are classified in [17].

Let $V$ be a real or complex vector space and $\mathfrak{g} \subset \mathfrak{g l}(V)$ a subalgebra. The space of skewsymmetric curvature tensors of type $\mathfrak{g}$ is defined as follows

$$
\overline{\mathcal{R}}(\mathfrak{g})=\left\{\begin{array}{c|c}
R \in \odot^{2} V^{*} \otimes \mathfrak{g} & \begin{array}{c}
R(X, Y) Z+R(Y, Z) X+R(Z, X) Y=0 \\
\text { for all } X, Y, Z \in V
\end{array}
\end{array}\right\} .
$$

The subalgebra $\mathfrak{g} \subset \mathfrak{g l}(V)$ is called a skew-Berger subalgebra if it is spanned by the images of the elements $R \in \overline{\mathcal{R}}(\mathfrak{g})$. Obviously $\overline{\mathcal{R}}(\mathfrak{g})=\mathcal{R}(\mathfrak{g} \subset \mathfrak{g l}(\Pi V))$ and $\mathfrak{g} \subset \mathfrak{g l}(V)$ is a skew-Berger algebra if and only if $\mathfrak{g} \subset \mathfrak{g l}(\Pi V)$ is a Berger superalgebra.

Let $\omega$ be the standard symplectic form on $V=\mathbb{R}^{2 m}$. A subalgebra $\mathfrak{g} \subset \mathfrak{s p}(2 m, \mathbb{R})$ is called weakly-irreducible if it does not preserve any proper non-degenerate subspace of $\mathbb{R}^{2 m}$. The $\mathrm{Wu}$ Theorem for supermanifolds implies the following statement. Let $\mathfrak{g} \subset \mathfrak{s p}(2 m, \mathbb{R})$ be an irreducible skew-Berger subalgebra, then there is a decomposition

$$
V=V_{0} \oplus V_{1} \oplus \cdots \oplus V_{r}
$$

into a direct sum of symplectic subspaces and a decomposition

$$
\mathfrak{g}=\mathfrak{g}_{1} \oplus \cdots \oplus \mathfrak{g}_{r}
$$

into a direct sum of ideals such that $\mathfrak{g}_{i}$ annihilates $V_{j}$ if $i \neq j$ and $\mathfrak{g}_{i} \subset \mathfrak{s p}\left(V_{i}\right)$ is a weaklyirreducible skew-Berger subalgebra.

Irreducible skew-Berger subalgebras $\mathfrak{g} \subset \mathfrak{g l}(n, \mathbb{C})$ are classified in [16]. Irreducible skew-Berger subalgebras $\mathfrak{g} \subset \mathfrak{s p}(2 m, \mathbb{R})$ are classified in [17].

Let $\mathfrak{g} \subset \mathfrak{s p}(2 m, \mathbb{R})=\mathfrak{s p}(V)$ be a reductive weakly-irreducible subalgebra. Suppose that $\mathfrak{g}$ is not irreducible. As in Section 2 we may show that $V$ is of the form $V=L \oplus L^{*}$, where $\mathfrak{g} \subset \mathfrak{g l}(L)$ is irreducible. If $\mathfrak{g} \subset \mathfrak{s p}(V)$ is a skew-Berger subalgebra, then $(\mathfrak{g} \subset \mathfrak{g l}(L))^{[1]} \neq\{0\}$, where

$$
\mathfrak{g}^{[1]}=\left\{\varphi \in L^{*} \otimes \mathfrak{g} \mid \varphi(x) y=-\varphi(y) x \text { for all } x, y \in L\right\}
$$

is the skew-symmetric prolongation of the subalgebra $\mathfrak{g} \subset \mathfrak{g l}(L)$. Irreducible subalgebras $\mathfrak{g} \subset$ $\mathfrak{g l}(L)$ with $\mathfrak{g}^{[1]} \neq 0$ are classified in [17].

Let $V$ be a complex or real vector space with a symplectic form $\omega$. Let $\mathfrak{g} \subset \mathfrak{s p}(V)$ be a subalgebra. The vector space

$$
\mathcal{P}_{\omega}(\mathfrak{g})=\left\{\begin{array}{c|c}
P \in V^{*} \otimes \mathfrak{g} & \omega(P(X) Y, Z)+\omega(P(Y) Z, X)+\omega(P(Z) X, Y)=0 \\
\text { for all } X, Y, Z \in V
\end{array}\right\}
$$

is called the space of skew-symmetric weak-curvature tensors of type $\mathfrak{g}$. A subalgebra $\mathfrak{g} \subset \mathfrak{s p}(V)$ is called a skew-symmetric weak-Berger algebra if $\mathfrak{g}$ is spanned by the images of the elements $P \in \mathcal{P}_{\omega}(\mathfrak{g})$. It is not hard to see that if $R \in \overline{\mathcal{R}}(\mathfrak{g})$ and $X \in V$ is fixed, then $R(\cdot, X) \in \mathcal{P}_{\omega}(\mathfrak{g})$. In particular, any skew-Berger algebra is a skew-symmetric weak-Berger algebra. The converse statement gives the following theorem.

Theorem 4 Let $\mathfrak{g} \subset \mathfrak{s p}(2 m, \mathbb{R})$ be an irreducible skew-symmetric weak-Berger subalgebra, then it is a skew-Berger subalgebra.

The proof of this theorem is a modified copy of the proof from [25] of Theorem 3 . 
If the representation $\mathfrak{g} \subset \mathfrak{s p}(2 m, \mathbb{R})$ is not absolutely irreducible, then $\mathcal{P}_{\omega}(\mathfrak{g})$ is isomorphic to $\left(\mathfrak{g}_{\mathbb{C}} \subset \mathfrak{g l}(m, \mathbb{C})\right)^{[1]}$ and the proof follows from [16, 17].

If the representation $\mathfrak{g} \subset \mathfrak{s p}(2 m, \mathbb{R})$ is absolutely irreducible, then we need a classification of irreducible skew-symmetric weak-Berger subalgebras $\mathfrak{g} \subset \mathfrak{s p}(2 m, \mathbb{C})$. It can be achieve in the same way as the classification of irreducible weak-Berger subalgebras $\mathfrak{g} \subset \mathfrak{s o}(n, \mathbb{C})$. In fact, in [25] it is obtained a necessary condition for an irreducible subalgebra $\mathfrak{g} \subset \mathfrak{s o}(n, \mathbb{C})$ to be a weak-Berger subalgebra, then there were classified all subalgebras $\mathfrak{g} \subset \mathfrak{g l}(n, \mathbb{C})$ satisfying this condition and the subalgebras $\mathfrak{g} \subset \mathfrak{s p}(2 m, \mathbb{C})$ were noted. It is easy to see that a skewsymmetric weak-Berger subalgebras $\mathfrak{g} \subset \mathfrak{s p}(2 m, \mathbb{C})$ satisfy the same necessary condition. Thus the proof follows immediately.

The spaces $\mathcal{P}_{\omega}(\mathfrak{g})$ for irreducible weak-Berger subalgebras $\mathfrak{g} \subset \mathfrak{s p}(2 n, \mathbb{R})$ can be found by methods of [14]. In particular, it is can be proved that if

$$
\mathfrak{g}=\mathfrak{g}^{1} \oplus \mathfrak{g}^{2} \subset \mathfrak{s p}\left(V^{1} \otimes V^{2}\right)=\mathfrak{s p}(V),
$$

where $\mathfrak{g}^{1} \subset \mathfrak{g l}\left(V^{1}\right)$ and $\mathfrak{g}^{2} \subset \mathfrak{g l}\left(V^{2}\right)$ are irreducible, then

$$
\mathcal{P}_{\omega}\left(\mathfrak{g}^{1} \subset \mathfrak{s p}(V)\right)=\mathcal{P}_{\omega}\left(\mathfrak{g}^{2} \subset \mathfrak{s p}(V)\right)=0,
$$

unless the complexification of $\mathfrak{g} \subset \mathfrak{s p}(V)$ coincides with

$$
\mathfrak{s o}(n, \mathbb{C}) \oplus \mathfrak{s l}(2, \mathbb{C}) \subset \mathfrak{s p}(2 n, \mathbb{C}) .
$$

For example, for $\mathfrak{s o}(n, \mathbb{R}) \oplus \mathfrak{s l}(2, \mathbb{R}) \subset \mathfrak{s p}(2 n, \mathbb{R})$ it holds

$$
\mathcal{P}_{\omega}(\mathfrak{s l}(2, \mathbb{R}) \subset \mathfrak{s p}(V))=0, \quad \mathcal{P}_{\omega}(\mathfrak{s o}(n, \mathbb{R}) \subset \mathfrak{s o}(V))=(\mathfrak{s o}(n, \mathbb{C}) \subset \mathfrak{s l}(n, \mathbb{C}))^{[1]}
$$

Let $\mathfrak{g} \subset \mathfrak{g l}(L)$ be an irreducible subalgebra. Then $\mathfrak{g}$ is a weakly-irreducible subalgebra of $\mathfrak{s p}\left(L \oplus L^{*}\right)=\mathfrak{s p}(2 m, \mathbb{R})$, where $m=\operatorname{dim} L$. Let $\omega$ be the natural symplectic form $L \oplus L^{*}$. It is easy to see that $P \in \mathcal{P}_{\omega}(\mathfrak{g})$ if and only if

$$
\left.\operatorname{pr}_{\mathfrak{g l}(L)} \circ P\right|_{L} \in(\mathfrak{g} \subset \mathfrak{g l}(L))^{[1]},\left.\quad \operatorname{pr}_{\mathfrak{g l}\left(L^{*}\right)} \circ P\right|_{L} \in\left(\mathfrak{g} \subset \mathfrak{g l}\left(L^{*}\right)\right)^{[1]}
$$

Thus if $\mathfrak{g} \subset \mathfrak{s p}\left(L \oplus L^{*}\right)$ is a skew-symmetric weak-Berger subalgebra, then $(\mathfrak{g} \subset \mathfrak{g l}(L))^{[1]} \neq\{0\}$ and $\mathfrak{g} \subset \mathfrak{g l}(L)$ is given in [17].

\section{$9 \quad$ Structure of the spaces $\mathcal{R}(\mathfrak{g})$}

Consider a subalgebra $\mathfrak{g} \subset \mathfrak{o} \mathfrak{s p}(p, q \mid 2 m)=\mathfrak{o s p}(V)$ and describe the space $\mathcal{R}(\mathfrak{g})$. Denote the supersymmetric metric on $V$ by $g$. It can be represented as the sum $g=\eta+\omega$, where $\eta$ is a pseudo-Euclidean metric of signature $(p, q)$ on $V_{\overline{0}}=\mathbb{R}^{p, q}$ and $\omega$ is a symplectic form on $\Pi V_{\overline{1}}=\mathbb{R}^{2 m}$. We identify $\mathfrak{o s p}(V)$ with $\Lambda^{2} V$, the element $X \wedge Y \in \mathfrak{o s p}(V)$ is defined by

$$
(X \wedge Y) Z=(-1)^{|Y||Z|} g(X, Z) Y-(-1)^{(|Y|+|Z|)|X|} g(Y, Z) X
$$

where $X, Y, Z \in V$ are homogeneous. Note that $\mathfrak{s o}(p, q) \simeq \Lambda^{2} V_{\overline{0}}$ and $\mathfrak{s p}(2 m, \mathbb{C}) \simeq \Lambda^{2} V_{\overline{1}}=$ $\odot^{2} \Pi V_{\overline{1}}$. From the Bianchi super identity it follows that any $R \in \mathcal{R}(\mathfrak{g})$ satisfies

$$
g(R(X, Y) Z, W)=(-1)^{(|X|+|Y|)(|Z|+|W|)} g(R(Z, W) X, Y)
$$

for all homogeneous $X, Y, Z, W \in V$. This means that $R: \Lambda^{2} V \rightarrow \mathfrak{g} \subset \Lambda^{2} V$ is a supersymmetric map. In particular, $R$ is zero on the orthogonal complement $\mathfrak{g}^{\perp} \subset \Lambda^{2} V$. 
First consider that space $\mathcal{R}(\mathfrak{g})_{\overline{0}}$. Let $R \in\left(\Lambda^{2} V^{*} \otimes \mathfrak{g}\right)_{\overline{0}}$. Define the following maps:

$$
\begin{aligned}
& A=\left.\operatorname{pr}_{\mathfrak{s o}(p, q)} \mathfrak{g}_{\overline{0}} \circ R\right|_{\Lambda^{2} V_{\overline{0}} \oplus \Lambda^{2} V_{\overline{1}}}: \Lambda^{2} V_{\overline{0}} \oplus \Lambda^{2} V_{\overline{1}} \rightarrow \operatorname{pr}_{\mathfrak{s o}(p, q)} \mathfrak{g}_{\overline{0}}, \\
& B=\left.\operatorname{pr}_{\mathbb{R}^{2 m *} \otimes \mathbb{R}^{p+q}} \mathfrak{g}_{\overline{1}} \circ R\right|_{V_{\overline{0}} \otimes V_{\overline{1}}}: V_{\overline{0}} \otimes V_{\overline{1}} \rightarrow \operatorname{pr}_{\mathbb{R}^{2 m *} \otimes \mathbb{R}^{p+q}} \mathfrak{g}_{\overline{1}}, \\
& C=\left.\operatorname{pr}_{\mathbb{R}^{p+q *} \otimes \mathbb{R}^{2 m}} \mathfrak{g}_{\overline{1}} \circ R\right|_{V_{\overline{0}} \otimes V_{\overline{1}}}: V_{\overline{0}} \otimes V_{\overline{1}} \rightarrow \operatorname{pr}_{\mathbb{R}^{p+q *} \otimes \mathbb{R}^{2 m}} \mathfrak{g}_{\overline{1}}, \\
& D=\left.\operatorname{pr}_{\mathfrak{s p}(2 m, \mathbb{R})} \mathfrak{g}_{\overline{0}} \circ R\right|_{\Lambda^{2} V_{\overline{0}} \oplus \Lambda^{2} V_{\overline{1}}}: \Lambda^{2} V_{\overline{0}} \oplus \Lambda^{2} V_{\overline{1}} \rightarrow \operatorname{pr}_{\mathfrak{s p}(2 m, \mathbb{R})} \mathfrak{g}_{\overline{0}} .
\end{aligned}
$$

In the definition of $B$ and $C$ we used the inclusion

$$
\mathfrak{o s p}(p, q \mid 2 m) \subset \mathfrak{g l}(p+q \mid 2 m, \mathbb{R})=\mathfrak{g l}(p+q, \mathbb{R}) \oplus \mathfrak{g l}(2 m, \mathbb{R}) \oplus \mathbb{R}^{2 m *} \otimes \mathbb{R}^{p+q} \oplus \mathbb{R}^{p+q *} \otimes \mathbb{R}^{2 m} .
$$

Since $R$ takes values in $\mathfrak{g} \subset \mathfrak{o s p}(p, q \mid 2 m)$, we obtain

$$
\omega(C(x, \xi) y, \delta)=-\eta(y, B(x, \xi) \delta)
$$

for all $x, y \in V_{\overline{0}}$ and $\xi, \delta \in V_{\overline{1}}$, i.e. the maps $B$ and $C$ define each other. Extend the maps $A, B, C, D$ to $\Lambda^{2} V$ mapping the natural complements to zero. Then $R=A+B+C+D$. In the matrix form we may write

$$
\begin{aligned}
& R(x, y)=-R(y, x)=\left(\begin{array}{cc}
A(x, y) & 0 \\
0 & D(x, y)
\end{array}\right), \quad R(\xi, \delta)=R(\delta, \xi)=\left(\begin{array}{cc}
A(\xi, \delta) & 0 \\
0 & D(\xi, \delta)
\end{array}\right), \\
& R(x, \xi)=-R(\xi, x)=\left(\begin{array}{cc}
0 & B(x, \xi) \\
C(x, \xi) & 0
\end{array}\right),
\end{aligned}
$$

where $x, y \in V_{\overline{0}}$ and $\xi, \delta \in V_{\overline{1}}$.

Writing down the Bianchi identity, we get that $R \in \mathcal{R}(\mathfrak{g})_{\overline{0}}$ if and only if the following conditions hold: $\left.A\right|_{\Lambda^{2} V_{\overline{0}}} \in \mathcal{R}\left(\operatorname{pr}_{\mathfrak{s o}(p, q)} \mathfrak{g}_{\overline{0}}\right),\left.D\right|_{\Lambda^{2} V_{\overline{1}}} \in \bar{R}\left(\operatorname{pr}_{\mathfrak{s p}(2 m, \mathbb{R})} \mathfrak{g}_{\overline{0}}\right)$,

$$
\begin{gathered}
D(x, y) \xi+C(y, \xi) x+C(\xi, x) y=0 \\
A(\xi, \delta) x-B(\delta, x) \xi+B(x, \xi) \delta=0
\end{gathered}
$$

for all $x, y \in V_{\overline{0}}$ and $\xi, \delta \in V_{\overline{1}}$.

Suppose that $R \in \mathcal{R}(\mathfrak{g})_{\overline{0}}$. Using (77), we get

$$
\begin{aligned}
& \omega(D(x, y) \xi, \delta)=\eta(A(\xi, \delta) x, y), \\
& \omega(C(x, \xi) y, \delta)=-\omega(C(y, \delta) x, \xi), \quad \eta(B(x, \xi) \delta, y)=-\eta(B(y, \delta) \xi, x)
\end{aligned}
$$

for all $x, y \in V_{\overline{0}}$ and $\xi, \delta \in V_{\overline{1}}$. In particular, we see that $\left.A\right|_{\Lambda^{2} V_{\overline{1}}}$ and $\left.D\right|_{\Lambda^{2} V_{\overline{0}}}$ define each other. The meanings of the restrictions (9) and (10) on $\left.A\right|_{\Lambda^{2} V_{\overline{1}}}$ and $\left.D\right|_{\Lambda^{2} V_{\overline{0}}}$ are not so clear. On the other hand, if representation of $\mathfrak{g}_{\overline{0}}$ is diagonal in $V_{\overline{0}} \oplus V_{\overline{1}}$ (by this we mean that the both representations of $\mathfrak{g}_{\overline{0}}$ on $V_{\overline{0}}$ and $V_{\overline{1}}$ are faithful), then $\left.A\right|_{\Lambda^{2} V_{\overline{1}}}$ and $\left.D\right|_{\Lambda^{2} V_{\overline{0}}}$ are given by $\left.D\right|_{\Lambda^{2} V_{\overline{1}}}$ and $\left.A\right|_{\Lambda^{2} V_{\overline{0}}}$, respectively. We will use this in many situations.

We turn now to the space $\mathcal{R}(\mathfrak{g})_{\overline{1}}$. Let $R \in\left(\Lambda^{2} V^{*} \otimes \mathfrak{g}\right)_{\overline{1}}$. Define the following maps:

$$
\begin{aligned}
A & =\left.\operatorname{pr}_{\mathfrak{s o}(p, q)} \mathfrak{g}_{\overline{0}} \circ R\right|_{V_{\overline{0}} \otimes V_{\overline{1}}}: V_{\overline{0}} \otimes V_{\overline{1}} \rightarrow \operatorname{pr}_{\mathfrak{s o}(p, q)} \mathfrak{g}_{\overline{0}}, \\
B & =\left.\operatorname{pr}_{\mathbb{R}^{2 m *} \otimes \mathbb{R}^{p+q}} \mathfrak{g}_{\overline{1}} \circ R\right|_{\Lambda^{2} V_{\overline{0}} \oplus \Lambda^{2} V_{\overline{1}}}: \Lambda^{2} V_{\overline{0}} \oplus \Lambda^{2} V_{\overline{1}} \rightarrow \operatorname{pr}_{\mathbb{R}^{2 m *} \otimes \mathbb{R}^{p+q}} \mathfrak{g}_{\overline{1}}, \\
C & =\left.\operatorname{pr}_{\mathbb{R}^{p+q *} \otimes \mathbb{R}^{2 m}} \mathfrak{g}_{\overline{1}} \circ R\right|_{\Lambda^{2} V_{\overline{0}} \oplus \Lambda^{2} V_{\overline{1}}}: \Lambda^{2} V_{\overline{0}} \oplus \Lambda^{2} V_{\overline{1}} \rightarrow \operatorname{pr}_{\mathbb{R}^{p+q *} \otimes \mathbb{R}^{2 m}} \mathfrak{g}_{\overline{1}}, \\
D & =\left.\operatorname{pr}_{\mathfrak{s p}(2 m, \mathbb{R})} \mathfrak{g}_{\overline{0}} \circ R\right|_{V_{\overline{0}} \otimes V_{\overline{1}}}: V_{\overline{0}} \otimes V_{\overline{1}} \rightarrow \operatorname{pr}_{\mathfrak{s p}(2 m, \mathbb{R})} \mathfrak{g}_{\overline{0}} .
\end{aligned}
$$

Since $R$ takes values in $\mathfrak{g} \subset \mathfrak{o s p}(p, q \mid 2 m)$, we obtain

$$
\omega(C(x, y) z, \xi)=-\eta(z, B(x, y) \xi), \quad \omega(C(\xi, \delta) z, \theta)=-\eta(z, B(\xi, \delta) \theta)
$$


for all $x, y, z \in V_{\overline{0}}$ and $\xi, \delta, \theta \in V_{\overline{1}}$. Thus the maps $B$ and $C$ define each other. Extend the maps $A, B, C, D$ to $\Lambda^{2} V$ mapping the natural complements to zero. Then $R=A+B+C+D$. In the matrix form we may write

$$
\begin{aligned}
& R(x, y)=-R(y, x)=\left(\begin{array}{cc}
0 & B(x, y) \\
C(x, y) & 0
\end{array}\right), \quad R(\xi, \delta)=R(\delta, \xi)=\left(\begin{array}{cc}
0 & B(\xi, \delta) \\
C(\xi, \delta) & 0
\end{array}\right), \\
& R(x, \xi)=-R(\xi, x)=\left(\begin{array}{cc}
A(x, \xi) & 0 \\
0 & D(x, \xi)
\end{array}\right),
\end{aligned}
$$

where $x, y \in V_{\overline{0}}$ and $\xi, \delta \in V_{\overline{1}}$. Writing down the Bianchi identity, we get that $R \in \mathcal{R}(\mathfrak{g})_{\overline{1}}$ if and only if the following conditions hold:

$$
\begin{aligned}
B(x, y) z+B(y, z) x+B(z, x) y & =0, \\
C(\xi, \delta) \theta+C(\delta, \theta) \xi+C(\theta, \xi) \delta & =0, \\
B(x, y) \xi+A(y, \xi) x+A(\xi, x) y & =0, \\
C(\xi, \delta) x-D(\delta, x) \xi+D(x, \xi) \delta & =0
\end{aligned}
$$

for all $x, y, z \in V_{\overline{0}}$ and $\xi, \delta, \theta \in V_{\overline{1}}$. Let us fix $\xi \in V_{\overline{1}}$. Using (14), we get

$$
\eta(B(x, y) \xi, z)+\eta(B(y, z) \xi, x)+\eta(B(z, x) \xi, y)=0
$$

for all $x, y, z \in V_{\overline{0}}$. Using this and (16), we conclude that for each fixed $\xi \in V_{\overline{1}}$ it holds $R(\cdot, \xi) \in \mathcal{P}_{\eta}\left(\operatorname{pr}_{\mathfrak{s o}(p, q)} \mathfrak{g}_{\overline{0}}\right)$. Similarly, for each $x \in V_{\overline{0}}$ it holds $R(\cdot, x) \in \mathcal{P}_{\omega}\left(\operatorname{pr}_{\mathfrak{s p}(2 m, \mathbb{R})} \mathfrak{g}_{\overline{0}}\right)$. This will be extremely useful especially in the case when the representation of $\mathfrak{g}_{\overline{0}}$ or of some ideal of $\mathfrak{g}_{\overline{0}}$ is diagonal in $V_{\overline{0}} \oplus V_{\overline{1}}$.

In [27] it is shown that

$$
\mathcal{R}(\mathfrak{o s p}(p, q \mid 2 m)) \simeq \odot^{2}\left(\Lambda^{2} V\right) / \Lambda^{4} V .
$$

The $\mathfrak{o s p}(p, q \mid 2 m)$-supermodule $\mathcal{R}(\mathfrak{o s p}(p, q \mid 2 m))$ is decomposed into the direct sum of three irreducible components. This generalizes the well-known decomposition of the $\mathfrak{s o}(p, q)$-module $\mathcal{R}(\mathfrak{s o}(p, q))$ [2] and defines the decomposition of the elements $R \in \mathcal{R}(\mathfrak{o s p}(p, q \mid 2 m))$ into the Weyl tensor, the trace-free part of the Ricci tensor and the scalar curvature.

Let us compare (18) with the above description. For that we consider $\mathcal{R}(\mathfrak{o s p}(p, q \mid 2 m))$ as an $\mathfrak{s o}(p, q) \oplus \mathfrak{s p}(2 m, \mathbb{R})$-module. It holds

$$
\begin{aligned}
\Lambda^{2} V= & \Lambda^{2} V_{\overline{0}} \oplus\left(V_{\overline{0}} \otimes V_{\overline{1}}\right) \oplus \Lambda^{2} V_{\overline{1}}, \\
\odot^{2}\left(\Lambda^{2} V\right)= & \odot^{2}\left(\Lambda^{2} V_{\overline{0}}\right) \bigoplus \odot^{2}\left(V_{\overline{0}} \otimes V_{\overline{1}}\right) \bigoplus \odot^{2}\left(\Lambda^{2} V_{\overline{1}}\right) \bigoplus \Lambda^{2} V_{\overline{0}} \otimes\left(V_{\overline{0}} \otimes V_{\overline{1}}\right) \\
& \bigoplus \Lambda^{2} V_{\overline{0}} \otimes \Lambda^{2} V_{\overline{1}} \bigoplus\left(V_{\overline{0}} \otimes V_{\overline{1}}\right) \otimes \Lambda^{2} V_{\overline{1}}, \\
\Lambda^{4} V= & \Lambda^{4} V_{\overline{0}} \bigoplus \Lambda^{3} V_{\overline{0}} \otimes V_{\overline{1}} \bigoplus \Lambda^{2} V_{\overline{0}} \otimes \Lambda^{2} V_{\overline{1}} \bigoplus V_{\overline{0}} \otimes \Lambda^{3} V_{\overline{1}} \bigoplus \Lambda^{4} V_{\overline{1}} .
\end{aligned}
$$

This implies

$$
\begin{aligned}
\mathcal{R}(\mathfrak{o s p}(p, q \mid 2 m))_{\overline{0}} \simeq & \left(\odot^{2}\left(\Lambda^{2} V\right) / \Lambda^{4} V\right)_{\overline{0}}=\odot^{2}\left(\Lambda^{2} V_{\overline{0}}\right) / \Lambda^{4} V_{\overline{0}} \\
& \bigoplus \odot^{2}\left(\Lambda^{2} V_{\overline{1}}\right) / \Lambda^{4} V_{\overline{1}} \bigoplus \odot^{2}\left(V_{\overline{0}} \oplus V_{\overline{1}}\right) .
\end{aligned}
$$

Note that $\mathcal{R}(\mathfrak{s o}(p, q)) \simeq \odot^{2}\left(\Lambda^{2} V_{\overline{0}}\right) / \Lambda^{4} V_{\overline{0}}$ [2]. Similarly,

$$
\overline{\mathcal{R}}(\mathfrak{s p}(2 m, \mathbb{R})) \simeq \odot^{2}\left(\odot^{2} \Pi V_{\overline{1}}\right) / \odot^{4} \Pi V_{\overline{1}}=\odot^{2}\left(\Lambda^{2} V_{\overline{1}}\right) / \Lambda^{4} V_{\overline{1}} .
$$

We conclude that

$$
\mathcal{R}(\mathfrak{o s p}(p, q \mid 2 m))_{\overline{0}} \simeq \mathcal{R}(\mathfrak{s o}(p, q)) \oplus \overline{\mathcal{R}}(\mathfrak{s p}(2 m, \mathbb{R})) \oplus \odot^{2}\left(V_{\overline{0}} \oplus V_{\overline{1}}\right)
$$


Let us describe this isomorphism. The inclusions $\mathcal{R}(\mathfrak{s o}(p, q)), \overline{\mathcal{R}}(\mathfrak{s p}(2 m, \mathbb{R})) \subset \mathcal{R}(\mathfrak{o s p}(p, q \mid 2 m))_{\overline{0}}$ are obvious. Let $B \in \odot^{2}\left(V_{\overline{0}} \oplus V_{\overline{1}}\right)=\Lambda^{2}\left(V_{\overline{0}} \oplus \Pi V_{\overline{1}}\right)$, i.e. $B$ is a skew-symmetric endomorphism of $V_{\overline{0}} \oplus \Pi V_{\overline{1}}$ with respect to the skew-symmetric form $\eta \otimes \omega$, and $B$ satisfies

$$
\eta \otimes \omega(B(x, \xi), y \otimes \delta)=-\eta \otimes \omega(B(y, \delta), x \otimes \xi) .
$$

Note that this corresponds to (12). The equation (8) defines the element $C$, the equations (91) and (10) defines the restrictions $\left.D\right|_{\Lambda^{2} V_{\overline{0}}}$ and $\left.A\right|_{\Lambda^{2} V_{\overline{1}}}$. Put in addition $\left.A\right|_{\Lambda^{2} V_{\overline{0}}}=0$ and $\left.D\right|_{\Lambda^{2} V_{\overline{1}}}=0$. We obtain and element $R \in \mathcal{R}(\mathfrak{o s p}(p, q \mid 2 m))_{\overline{0}}$. This defines the inclusion $\odot^{2}\left(V_{\overline{0}} \oplus V_{\overline{1}}\right) \subset$ $\mathcal{R}(\mathfrak{o s p}(p, q \mid 2 m)) \overline{0}$.

Next,

$$
\mathcal{R}(\mathfrak{o s p}(p, q \mid 2 m))_{\overline{1}} \simeq V_{\overline{1}} \otimes\left(\Lambda^{2} V_{\overline{0}} \otimes V_{\overline{0}}\right) / \Lambda^{3} V_{\overline{0}} \bigoplus V_{\overline{0}} \otimes\left(\Lambda^{2} V_{\overline{1}} \otimes V_{\overline{1}}\right) / \Lambda^{3} V_{\overline{1}}
$$

It holds $\mathcal{P}_{\eta}(\mathfrak{s o}(p, q)) \simeq\left(\Lambda^{2} V_{\overline{0}} \otimes V_{\overline{0}}\right) / \Lambda^{3} V_{\overline{0}}\left[14\right.$. Similarly, $\mathcal{P}_{\omega}(\mathfrak{s p}(2 m, \mathbb{R})) \simeq\left(\odot^{2} \Pi V_{\overline{1}} \otimes \Pi V_{\overline{1}}\right) / \odot^{3}$ $\Pi V_{\overline{1}}$. We obtain

$$
\mathcal{R}(\mathfrak{o s p}(p, q \mid 2 m))_{\overline{1}} \simeq \mathcal{P}_{\eta}(\mathfrak{s o}(p, q)) \otimes V_{\overline{1}} \bigoplus \Pi \mathcal{P}_{\omega}(\mathfrak{s p}(2 m, \mathbb{R})) \otimes V_{\overline{0}}
$$

Let $P \in \mathcal{P}_{\eta}(\mathfrak{s o}(p, q))$ and $\zeta \in V_{\overline{0}}$ be fixed. Define $R \in \mathcal{R}(\mathfrak{o s p}(p, q \mid 2 m))_{\overline{1}}$ by putting

$$
A(x, \delta)=\omega(\delta, \zeta) P(x), D(x, \delta)=0, B(x, y) \xi=\omega(\zeta, \xi)(P(x) y-P(y) x), B(\xi, \delta)=0 .
$$

In the same way any elements $P \otimes x \in \mathcal{P}_{\omega}(\mathfrak{s p}(2 m, \mathbb{R})) \otimes V_{\overline{0}}$ define an $R \in \mathcal{R}(\mathfrak{o s p}(p, q \mid 2 m))_{\overline{1}}$. This gives the exact form of the obtained isomorphism.

Information about the spaces $\mathcal{R}(\mathfrak{g})$ for some Lie superalgebras $\mathfrak{g} \subset \mathfrak{g l}(n \mid k)$ not contained in $\mathfrak{o s p}(p, q \mid 2 m)$ can be found in [27, 32$]$.

\section{Adjoint representations of simple Lie superalgebras}

Proposition 6 Let $\mathfrak{g}$ be a simple (real or complex) Lie superalgebra admitting an even nondegenerate $\mathfrak{g}$-invariant bilinear supersymmetric form, i.e. such that the adjoint representation of $\mathfrak{g}$ is orthosymplectic. Then $\mathcal{R}(\mathfrak{g})=\mathcal{R}(\mathfrak{g})_{\overline{0}}$ is one-dimensional and it is spanned by the Lie superbrackets of $\mathfrak{g}$.

Proof. First of all, from the Jacobi super identity it follows that $[\cdot, \cdot] \in \mathcal{R}(\mathfrak{g})_{\overline{0}}$ for each simple Lie superalgebra. Note that the representation of $\mathfrak{g}_{\overline{0}}$ is diagonal in $\mathfrak{g}_{\overline{0}} \oplus \mathfrak{g}_{\overline{1}}$ (up to the center of $\mathfrak{g}_{\overline{0}}$, which does not play a role).

First we prove that $\mathcal{R}(\mathfrak{g})_{\overline{1}}=0$. Suppose that $\mathfrak{g}_{\overline{0}}$ contains at least two simple ideals $\mathfrak{h}_{1}$ and $\mathfrak{h}_{2}$. Let $R \in \mathcal{R}(\mathfrak{g})_{\overline{1}}$, then for each fixed $\xi \in \mathfrak{g}_{\overline{1}}$ and any $x \in \mathfrak{h}_{1}$ we have $R(x, \xi) \in \mathfrak{h}_{1}$. On the other hand, for each fixed $x \in \mathfrak{h}_{1}$ we have $\operatorname{pr}_{\mathfrak{g}_{\overline{0}}} \circ R(x, \cdot) \in \mathcal{P}_{\omega}\left(\operatorname{pr}_{\mathfrak{s p}\left(\mathfrak{g}_{\overline{1}}\right)} \mathfrak{g}_{\overline{0}}\right)$, but the $\mathfrak{g}_{\overline{0}}$-module $\mathfrak{g}_{\overline{1}}$ is a tensor product of irreducible representations of simple ideals in $\mathfrak{g}_{\overline{0}}$ (if $\mathfrak{g}$ is of type I) and it is a direct sum of two such representations (if $\mathfrak{g}$ is of type II). This and Section 8 show that $\operatorname{pr}_{\mathfrak{g}_{\overline{0}}} \circ R(x, \cdot)$ can not take values in a one simple ideal of $\mathfrak{g}_{\overline{0}}$ (unless $\mathfrak{g}_{\overline{0}}$ or its complexification coincides with $\mathfrak{s o}(n, \mathbb{C}) \oplus \mathfrak{s l}(2, \mathbb{C})$, i.e. if $\mathfrak{g}=\mathfrak{o} \mathfrak{s p}(p, q \mid 2)$ or $\mathfrak{g}=\mathfrak{o} \mathfrak{s p}(p \mid 2, \mathbb{C})$, these cases can be considered in the same way as $\mathfrak{g}=\mathfrak{o s p}(1 \mid 2 m, \mathbb{R})$ below and we get $\left.\mathcal{R}(\mathfrak{g})_{\overline{1}}=0\right)$. We have $\operatorname{pr}_{\mathfrak{g}_{\overline{0}}} \circ R(x, \cdot)=0$ for all $x \in \mathfrak{h}_{1}$. Similarly, $\operatorname{pr}_{\mathfrak{g}_{\overline{0}}} \circ R(x, \cdot)=0$ for all $x \in \mathfrak{h}_{2}$ and $\operatorname{pr}_{\mathfrak{g}_{\overline{0}}} \circ R(x, \cdot)=0$ for all $x \in \mathfrak{g}_{\overline{0}}$, i.e. $R=0$.

Suppose that the semi-simple part of $\mathfrak{g}_{\overline{0}}$ is simple, then $\mathfrak{g}=\mathfrak{o} \mathfrak{s p}(1 \mid 2 m, \mathbb{F})$ or $\mathfrak{g}=\mathfrak{o} \mathfrak{s} \mathfrak{p}(2 \mid 2 m, \mathbb{F})$, $\mathbb{F}=\mathbb{R}$ or $\mathbb{C}$ (for other simple $\mathfrak{g}$ such that the semi-simple part of $\mathfrak{g}_{\overline{0}}$ is simple, the adjoint representation of $\mathfrak{g}$ is not orthosymplectic). 
Consider the case $\mathfrak{g}=\mathfrak{o} \mathfrak{s p}(1 \mid 2 m, \mathbb{F})$, the case $\mathfrak{g}=\mathfrak{o} \mathfrak{s p}(2 \mid 2 m, \mathbb{F})$ is similar. Since the complexification of the adjoint representation of $\mathfrak{g}=\mathfrak{o s p}(1 \mid 2 m, \mathbb{R})$ is irreducible, it is enough to consider the adjoint representation of $\mathfrak{g}=\mathfrak{o} \mathfrak{s p}(1 \mid 2 m, \mathbb{C})$. Let $R \in \mathcal{R}(\mathfrak{g})_{\overline{1}}$. Then for each $x \in \mathfrak{g}_{\overline{0}}=\mathfrak{s p}(2 m, \mathbb{C})$ it holds $\operatorname{pr}_{\mathfrak{g}_{0}} \circ R(x, \cdot) \in \mathcal{P}_{\omega}(\mathfrak{s p}(2 m, \mathbb{C}))$, and for each $\xi \in \mathfrak{g}_{\overline{1}}=\mathbb{C}^{2 m}$ it holds $\operatorname{pr}_{\mathfrak{g}_{\overline{0}}} \circ R(\cdot, \xi) \in \mathcal{P}_{\eta}\left(\operatorname{ad}_{\mathfrak{s p}(2 m, \mathbb{C})}\right)$. That is $R(\mathfrak{g})_{\overline{1}}$ is contained in the diagonal form in the $\mathfrak{s p}(2 m, \mathbb{C})$-module

$$
\left(\mathbb{C}^{2 m} \otimes \mathcal{P}_{\eta}\left(\operatorname{ad}_{\mathfrak{s p}(2 m, \mathbb{C})}\right)\right) \oplus\left(\mathfrak{s p}(2 m, \mathbb{C}) \otimes \mathcal{P}_{\omega}(\mathfrak{s p}(2 m, \mathbb{C}))\right)
$$

Suppose that $m \geq 2$. In [14] we prove that $\mathcal{P}_{\eta}\left(\operatorname{ad}_{\mathfrak{s p}(2 m, \mathbb{C})}\right) \simeq \mathfrak{s p}(2 m, \mathbb{C})$ and any $P \in \mathcal{P}_{\eta}\left(\operatorname{ad}_{\mathfrak{s p}(2 m, \mathbb{C})}\right)$ is of the form $P(\cdot)=[x, \cdot]$, where $x \in \mathfrak{s p}(2 m, \mathbb{C})$. Note that $\mathcal{P}_{\omega}(\mathfrak{s p}(2 m, \mathbb{C}))$ contains a submodule isomorphic to $\mathbb{C}^{2 m}$ and any element $P$ of this module is of the form $P(\cdot)=\xi \odot \cdot$ for some $\xi \in \mathbb{C}^{2 m}$, here for $\xi, \delta \in \mathbb{C}^{2 m}$ the element $\xi \odot \delta \in \mathfrak{s p}(2 m, \mathbb{C})$ is defined by

$$
(\xi \odot \delta) \theta=\omega(\xi, \theta) \delta+\omega(\delta, \theta) \xi .
$$

We conclude that $R(\mathfrak{g})_{\overline{1}}$ is contained in the diagonal form in the $\mathfrak{s p}(2 m, \mathbb{C})$-module

$$
\left(\mathbb{C}^{2 m} \otimes \mathfrak{s p}(2 m, \mathbb{C})\right) \oplus\left(\mathfrak{s p}(2 m, \mathbb{C}) \otimes \mathbb{C}^{2 m}\right) .
$$

Moreover for each $R \in R(\mathfrak{g})_{\overline{1}}$ there exist linear maps $\varphi: \mathbb{C}^{2 m} \rightarrow \mathfrak{s p}(2 m, \mathbb{C})$ and $\psi: \mathfrak{s p}(2 m, \mathbb{C}) \rightarrow$ $\mathbb{C}^{2 m}$ such that $R(x, \xi)=[\varphi(\xi), x]=\psi(x) \odot \xi$ for all $\xi \in \mathbb{C}^{2 m}$ and $x \in \mathfrak{s p}(2 m, \mathbb{C})$. Since $R(\mathfrak{g})_{\overline{1}}$ is an $\mathfrak{s p}(2 m, \mathbb{C})$-module, $\varphi$ and $\psi$ are proportional as the elements of $\mathbb{C}^{2 m} \otimes \mathfrak{s p}(2 m, \mathbb{C})$. To show that the equation $[\varphi(\xi), x]=\psi(x) \odot \xi$ for all $\xi \in \mathbb{C}^{2 m}$ and $x \in \mathfrak{s p}(2 m, \mathbb{C})$ has only the trivial solution it is enough to decompose the $\mathfrak{s p}(2 m, \mathbb{C})$-module $\mathbb{C}^{2 m} \otimes \mathfrak{s p}(2 m, \mathbb{C})$ into the direct some of irreducible components and to check this equation for a non-zero representative of each component. We have

$$
\mathbb{C}^{2 m} \otimes \mathfrak{s p}(2 m, \mathbb{C})=V_{3 \pi_{1}} \oplus V_{\pi_{1}+\pi_{2}} \oplus \mathbb{C}^{2 m} .
$$

Let $\left(\xi_{\alpha}\right)_{\alpha=-m, \ldots,-1,1, \ldots, m}$ be the standard basis of $\mathbb{C}^{2 m}$, i.e. $\omega\left(\xi_{\alpha}, \xi_{\beta}\right)=\delta_{\alpha,-\beta}$. Then $\mathfrak{s p}(2 m, \mathbb{C})$ is spanned be the elements of the form $\xi_{\alpha} \odot \xi_{\beta}$. Let

$$
\varphi=c \psi=\xi_{1} \otimes \xi_{1} \odot \xi_{1} \in V_{3 \pi_{1}} .
$$

Substituting to the equation $\xi=\xi_{-1}$ and $x=\xi_{-1} \odot \xi_{1}$, we get

$$
0=\left[\xi_{1} \odot \xi_{1}, \xi_{-1} \odot \xi_{1}\right] .
$$

On the other hand,

$$
\left[\xi_{1} \odot \xi_{1}, \xi_{-1} \odot \xi_{1}\right]=2 \xi_{1} \odot \xi_{1} \neq 0 .
$$

Hence $\varphi=c \psi=\xi_{1} \otimes \xi_{1} \odot \xi_{1}$ is not a solution of the equation. Similarly, taking

$$
\varphi=c \psi=\sum_{\alpha} \xi_{\alpha} \otimes \xi_{-\alpha} \odot \xi_{1} \in \mathbb{C}^{2 m}, \quad \xi=\xi_{1}, \quad x=\xi_{1} \odot \xi_{1},
$$

we get that $\varphi=c \psi=\sum_{\alpha} \xi_{\alpha} \otimes \xi_{-\alpha} \odot \xi_{1}$ is not a solution of the equation. Finally, the $\mathfrak{s p}(2 m, \mathbb{C})$ module $\mathbb{C}^{2 m} \otimes \mathfrak{s p}(2 m, \mathbb{C})$ contains the weight space of the weight $\pi_{1}+\pi_{2}$ of dimension 2 and this space consists of the vectors

$$
c_{1} \xi_{1} \otimes \xi_{1} \odot \xi_{2}+c_{2} \xi_{2} \otimes \xi_{1} \odot \xi_{1}, \quad c_{1}, c_{2} \in \mathbb{C} .
$$

Let $\varphi=c \psi$ be equal to such vector. Taking $\xi=\xi_{-1}$ and $x=\xi_{-2} \odot \xi_{1}$, we get $c_{1}=0$; taking $\xi=\xi_{-2}$ and $x=\xi_{-1} \odot \xi_{2}$, we get $c_{2}=0$. Thus we may conclude that $\mathcal{R}(\mathfrak{g})_{\overline{1}}=0$. If $m=1$, then $\mathbb{C}^{2} \otimes \mathfrak{s p}(2, \mathbb{C})=V_{3 \pi_{1}} \oplus \mathbb{C}^{2}$. It is not hard to see that $\mathcal{P}_{\omega}(\mathfrak{s p}(2, \mathbb{C}))=\mathbb{C}^{2}$. And the further proof is the same. 
Next we prove that $\mathcal{R}(\mathfrak{g})_{\overline{0}}$ is one-dimensional.

Let $R \in \mathcal{R}(\mathfrak{g})_{\overline{0}}$ be given as above by the linear maps $A, B, C, D$. We have seen that $\left.A\right|_{\Lambda^{2} V_{\overline{0}}}$ (or $\left.D\right|_{\Lambda^{2} V_{\overline{1}}}$ ) defines uniquely $A$ and $D$. We claim that it defines the whole $R$. Indeed, suppose that $A=0$ and $D=0$. Let $\xi, \delta \in \mathfrak{g}_{\overline{1}}$ and $x \in \mathfrak{g}_{\overline{0}}$. We have $\xi \cdot R(x, \delta)=[\xi, R(x, \delta)]$. If $R(x, \delta) \neq 0$, then since $\mathfrak{g}$ is simple, there exists a $\xi$ such that $\xi \cdot R(x, \delta) \neq 0$. On the other hand, $\xi \cdot R \in \mathcal{R}(\mathfrak{g})_{\overline{1}}=0$ and we get a contradiction, this proves the claim.

If the semi-simple part of $\mathfrak{g}_{\overline{0}}$ is simple, then $\left.A\right|_{\Lambda^{2} V_{\overline{0}}}$ being an element in $\mathcal{R}\left(\operatorname{ad}_{\mathfrak{g}_{\overline{0}}}\right)$ is proportional to the Lie brackets in $\mathfrak{g}_{\overline{0}}$ [34]. Since $\left.A\right|_{\Lambda^{2} V_{\overline{0}}}$ defines uniquely $R \in \mathcal{R}(\mathfrak{g})_{\overline{0}}$, we get that $\mathcal{R}(\mathfrak{g})_{\overline{0}}$ is one-dimensional.

Suppose that $\mathfrak{g}$ is of type I and the semi-simple part of $\mathfrak{g}_{\overline{0}}$ is not simple, then $\overline{\mathcal{R}}\left(\mathfrak{g}_{\overline{0}} \subset \mathfrak{s p}\left(\mathfrak{g}_{\overline{1}}\right)\right)$ is one-dimensional (Section 8). Hence $\left.D\right|_{\Lambda^{2} V_{\overline{1}}}$ belongs to a one-dimensional space. Since $\left.D\right|_{\Lambda^{2} V_{\overline{1}}}$ defines uniquely $R \in \mathcal{R}(\mathfrak{g})_{\overline{0}}$, we get that $\mathcal{R}(\mathfrak{g})_{\overline{0}}$ is one-dimensional.

Finally suppose that $\mathfrak{g}$ is of type II and the semi-simple part of $\mathfrak{g}_{\overline{0}}$ is not simple, i.e. $\mathfrak{g}_{\overline{0}}=$ $\mathfrak{h}_{1} \oplus \mathfrak{h}_{2} \oplus \mathfrak{z}$. Then obviously $\left.A\right|_{\mathfrak{h}_{1} \otimes \mathfrak{h}_{2}}=0,\left.A\right|_{\Lambda^{2} \mathfrak{h}_{1}}=c_{1}[\cdot, \cdot]_{\mathfrak{h}_{1}}$, and $\left.A\right|_{\Lambda^{2} \mathfrak{h}_{2}}=c_{2}[\cdot, \cdot]_{\mathfrak{h}_{2}}$ are annihilated by $\mathfrak{g}_{\overline{0}}$. Then $\left.D\right|_{\Lambda^{2} V_{\overline{1}}}$ belongs to a two-dimensional space annihilated by $\mathfrak{g}_{\overline{0}}$. On the other hand, $\mathfrak{g}_{\overline{0}}$ may annihilate only a one-dimensional subspace in $\overline{\mathcal{R}}\left(\mathfrak{g}_{\overline{0}} \subset \mathfrak{s} \mathfrak{p}\left(\mathfrak{g}_{\overline{1}}\right)\right)$. Hence there exists a $c \in \mathbb{C}$ such that for each $R$ it holds $c_{1}=c c_{2}$. Thus $\mathcal{R}(\mathfrak{g})_{\overline{0}}$ is one-dimensional. The proposition is proved.

\section{Proof of Theorem 2}

Lemma 1 Let $\mathfrak{g} \subset \mathfrak{o s p}(p, q \mid 2 m)$ be an irreducible subalgebra of the form (1). If $\mathcal{R}(\mathfrak{g})_{\overline{1}}=0$, then $\mathcal{R}(\mathfrak{g})$ is a trivial $\mathfrak{g}$-module.

Proof. We have $\left(\mathfrak{g}_{i}\right)_{\overline{1}} \cdot \mathcal{R}(\mathfrak{g})_{\overline{0}} \subset \mathcal{R}(\mathfrak{g})_{\overline{1}}=0$. Since each $\mathfrak{g}_{i}$ is simple of classical type, it holds $\left(\mathfrak{g}_{i}\right)_{\overline{0}}=\left[\left(\mathfrak{g}_{i}\right)_{\overline{1}},\left(\mathfrak{g}_{i}\right)_{\overline{1}}\right]$. Consequently, $\left(\mathfrak{g}_{i}\right)_{\overline{0}} \cdot \mathcal{R}(\mathfrak{g})_{\overline{0}}=0$. Suppose that $\mathfrak{z} \neq 0$. Since $\mathfrak{o s p}(p, q \mid 2 m)_{\overline{0}} \cap \mathfrak{q}(2 m, \mathbb{R})=0$, by the Schur Lemma $\mathfrak{z}=\mathbb{R} J$, where $J$ is an even complex structure on $V$. It is not hard to see that $J \cdot \mathcal{R}(\mathfrak{g})=0$.

First we consider case by case simple real Lie superalgebras $\mathfrak{g}$ of classical type (they are classified in [31] and we list them for the convenience in [17]). For each $\mathfrak{g}$ we find all irreducible representations $\mathfrak{g} \subset \mathfrak{o s p}(p, q \mid 2 m)=\mathfrak{o} \mathfrak{s p}(V)$ such that $\mathfrak{g}$ is a non-symmetric Berger supersubalgebra. We explain the way of the considerations and then give several examples demonstrating this proof.

We begin with the case when $\mathfrak{g}_{\overline{0}}$ is of the form $\mathfrak{h}_{1} \oplus \mathfrak{h}_{2} \oplus \mathfrak{z}$, where $\mathfrak{h}_{1}$ and $\mathfrak{h}_{2}$ are simple and $\mathfrak{z}$ is trivial or one-dimensional. If $\mathfrak{g}$ is of type I, i.e. the $\mathfrak{g}_{\overline{0}}$-module $\mathfrak{g}_{\overline{1}}$ is irreducible, then $\mathfrak{g}_{\overline{1}}$ is of the form $W_{1} \otimes W_{2}, \mathfrak{h}_{1} \subset \mathfrak{s o}\left(W_{1}\right)$ and $\mathfrak{h}_{2} \subset \mathfrak{s p}\left(W_{2}\right)$ are irreducible. If $\mathfrak{g}$ is of type II, i.e. the $\mathfrak{g}_{\overline{0}}$-module $\mathfrak{g}_{\overline{1}}$ is of the form $\mathfrak{g}_{-1} \oplus \mathfrak{g}_{1}$, where $\mathfrak{g}_{-1}$ and $\mathfrak{g}_{1}$ are irreducible $\mathfrak{g}_{\overline{0}}$-modules, then there are two vector spaces $U_{1}$ and $U_{2}$ such that $\mathfrak{h}_{1} \subset \mathfrak{g l}\left(U_{1}\right), \mathfrak{h}_{2} \subset \mathfrak{g l}\left(U_{2}\right)$ are irreducible, $\mathfrak{g}_{-1}=U_{1}^{*} \otimes U_{2}$, and $\mathfrak{g}_{1}=U_{2}^{*} \otimes U_{1}$.

Consider several cases:

Case a. $\mathfrak{h}_{1}$ annihilates $V_{\overline{1}}$. Suppose that $\mathfrak{g}$ is of type I. Since the inclusion $i: \mathfrak{g} \hookrightarrow \mathfrak{o} \mathfrak{s} \mathfrak{p}(p, q \mid 2 m)$ is a Lie superalgebra homomorphism, the restriction

$$
\left.i\right|_{\mathfrak{g}_{\overline{1}}}: \mathfrak{g}_{\overline{1}}=W_{1} \otimes W_{2} \rightarrow \mathfrak{o s p}(V)_{\overline{1}}=V_{\overline{0}} \otimes V_{\overline{1}}
$$

is $\mathfrak{g}_{\overline{0}}$-equivariant. In particular, it is $\mathfrak{h}_{1}$-equivariant. Since $\mathfrak{h}_{1}$ annihilates $W_{2}$ and $V_{\overline{1}}$, we conclude that $V_{\overline{0}}$ is a direct sum of $\mathfrak{h}_{1}$-submodules isomorphic to $W_{1}$ and of an $\mathfrak{h}_{1}$-trivial submodule. Similarly, if $\mathfrak{g}$ is of type II, then $V_{\overline{0}}$ is a direct sum of $\mathfrak{h}_{1}$-submodules isomorphic to $U_{1} \oplus U_{1}^{*}$ and of an $\mathfrak{h}_{1}$-trivial submodule. 
Under the current assumption we have three cases:

Case a.1. $\mathfrak{h}_{2}$ annihilates $V_{\overline{0}}$. Suppose that $\mathfrak{g}$ is of type I. By the above arguments, $V_{\overline{1}}$ is a direct sum of $\mathfrak{h}_{2}$-submodules isomorphic to $W_{2}$ and of an $\mathfrak{h}_{2}$-trivial submodule. From Sections 7 and 8 we read that if $V_{\overline{0}}$ contains more then one $\mathfrak{h}_{1}$-submodule isomorphic to $W_{1}$ and if $V_{\overline{1}}$ contains more then one $\mathfrak{h}_{2}$-submodule isomorphic to $W_{2}$, then $\mathcal{P}_{\eta}\left(\mathfrak{h}_{1} \subset \mathfrak{s o}\left(V_{\overline{0}}\right)\right)=0$ and $\mathcal{P}_{\omega}\left(\mathfrak{h}_{2} \subset\right.$ $\left.\mathfrak{s o}\left(V_{\overline{1}}\right)\right)=0$. Consequently, $\mathcal{R}(\mathfrak{g})_{\overline{1}}=0$ and $\mathfrak{g}$ is symmetric. Thus either $V_{\overline{0}}$ contains exactly one $\mathfrak{h}_{1}$-submodule isomorphic to $W_{1}$, or $V_{\overline{1}}$ contains exactly one $\mathfrak{h}_{2}$-submodule isomorphic to $W_{2}$. Similarly, if $\mathfrak{g}$ is of type II, then $V_{\overline{0}}$ contains exactly one $\mathfrak{h}_{1}$-submodule isomorphic to $U_{1} \oplus U_{1}^{*}$ or $V_{\overline{1}}$ contains exactly one $\mathfrak{h}_{2}$-submodule isomorphic to $U_{2} \oplus U_{2}^{*}$. Next we check when such representation of $\mathfrak{g}$ exists. For this we may pass to the complexification of $\mathfrak{g}$ and of its representation. If the resulting representation is not irreducible, we take one of its irreducible components. Note that the type of $\mathfrak{g}$ may change.

Let $\mathfrak{g}$ be of type I. Suppose, for instance, that $V_{\overline{1}}$ contains exactly one $\mathfrak{h}_{2}$-submodule isomorphic to $W_{2}$. Since $\mathfrak{g}_{\overline{1}} \otimes W_{2}$ contains only one submodule annihilated by $\mathfrak{h}_{2}$ and isomorphic to $W_{1}$ as the $\mathfrak{h}_{1}$-module and since the representation of $\mathfrak{g}_{\overline{1}}$ on $V$ is $\mathfrak{g}_{\overline{0}}$-equivariant, $\mathfrak{g}$ preserves the vector supersubspace $\left(\mathfrak{g}_{\overline{1}} \cdot V_{\overline{1}}\right) \oplus V_{\overline{1}} \subset V$ and its even part contains only one $\mathfrak{h}_{1}$-submodule isomorphic to $W_{1}$. From the irreducibility of $V$ it follows that $V_{\overline{0}}$ contains exactly one $\mathfrak{h}_{1}$-submodule isomorphic to $W_{1}$ and $V_{\overline{1}}$ contains exactly one $\mathfrak{h}_{2}$-submodule isomorphic to $W_{2}$. Suppose that $V$ contains a non-trivial vector $v$ annihilated by $\mathfrak{g}_{\overline{0}}$. Then the homogeneous components of $v$ are also annihilated by $\mathfrak{g}_{\overline{0}}$ and we may assume that $v$ is homogeneous. Suppose that $v \in V_{\overline{0}}$. Consider the map from $\mathfrak{g}_{\overline{1}}$ to $V_{\overline{1}}$ sending $\varphi \in \mathfrak{g}_{\overline{1}}$ to $\varphi v$. This map is $\mathfrak{g}_{\overline{0}}$-equivariant and since the $\mathfrak{g}_{\overline{0}}$-modules $\mathfrak{g}_{\overline{1}}$ and $V_{\overline{1}}$ are not isomorphic, this map is zero. This shows that $\mathbb{R} v \subset V$ is an invariant subspace and it must be trivial. Thus we get that $V_{\overline{0}}=W_{1}$ and $V_{\overline{1}}=W_{2}$. Such a representation of $\mathfrak{g}$ is either the identity one, or it does not exist. Let $\mathfrak{g}$ be of type II. By the similar arguments we get that $V_{\overline{0}}=U_{1}$ and $V_{\overline{1}}=U_{2}$. This happens e.g. for the complexification of the identity representation of $\mathfrak{s u}\left(p_{0}, q_{0} \mid p_{1}, q_{1}\right)$.

Case a.2. $\mathfrak{h}_{2}$ annihilates $V_{\overline{1}}$. In this case $\mathfrak{h}_{1} \oplus \mathfrak{h}_{2}$ annihilates $V_{\overline{1}}$. Let $U \subset V_{\overline{0}}$ be an irreducible $\mathfrak{h}_{1} \oplus \mathfrak{h}_{2}$-module. Since $U$ is not $\mathfrak{g}$-invariant, $\mathfrak{g}_{\overline{1}} \cdot U \neq 0$. On the other hand, $\mathfrak{g}_{\overline{1}} \cdot U \subset V_{1}$ is annihilated by $\mathfrak{h}_{1} \oplus \mathfrak{h}_{2}$, i.e. $\mathfrak{g}_{\overline{1}} \otimes U$ contains a one-dimensional subspace annihilated by $\mathfrak{h}_{1} \oplus \mathfrak{h}_{2}$. This may happens only if $U \simeq \mathfrak{g}_{\overline{1}}$ (if $\mathfrak{g}$ is of type I), or if $U$ is isomorphic either to $\mathfrak{g}_{-1}$, or to $\mathfrak{g}_{1}$ (if $\mathfrak{g}$ is of type II). We show that such representations do not exist.

Case a.3. The representation of $\mathfrak{h}_{2}$ is diagonal in $V_{\overline{0}} \oplus V_{\overline{1}}$. We may decompose $V_{\overline{0}}$ as the direct sum $V_{\overline{0}}=L_{1} \oplus L_{2} \oplus L_{3} \oplus L_{4}$ such that $\mathfrak{h}_{1} \oplus \mathfrak{h}_{2}$ annihilates $L_{4}, \mathfrak{h}_{1}$ annihilates $L_{2}, \mathfrak{h}_{2}$ annihilates $L_{1}$, $L_{1}$ is a direct sum of $\mathfrak{h}_{1}$-submodules isomorphic to $W_{1}$ (resp. $\left.U_{1} \oplus U_{1}^{*}\right), L_{2}$ is an $\mathfrak{h}_{2}$-submodule, and $L_{3}$ is an $\mathfrak{h}_{1} \oplus \mathfrak{h}_{2}$-submodule (such that each irreducible component of $L_{3}$ is faithful for both $\mathfrak{h}_{1}$ and $\left.\mathfrak{h}_{2}\right)$. If $L_{3} \neq 0$, then from Section 7 it follows that $\mathcal{P}_{\eta}\left(\operatorname{pr}_{\mathfrak{s o}\left(L_{1} \oplus L_{2}\right)} \mathfrak{g}_{\overline{0}}\right)=0$ and this implies $\mathcal{R}(\mathfrak{g})_{\overline{1}}=0$. Thus, $L_{3}=0$. Since $\mathfrak{g}$ is a Berger algebra, one of the following holds:

1. There exists an $R \in \mathcal{R}(\mathfrak{g})_{\overline{0}}$ such that for some $x, y \in L_{2}$ it holds $0 \neq R(x, y) \in \mathfrak{h}_{2}$. Expressing $R$ in terms of the maps $A, B, C, D$ as above, we get $\left.A\right|_{\Lambda^{2} L_{2}} \neq 0$ and $\left.D\right|_{\Lambda^{2} L_{2}} \neq 0$. Hence $\left.A\right|_{\Lambda^{2} V_{\overline{1}}} \neq 0$ and $\left.D\right|_{\Lambda^{2} V_{\overline{1}}} \neq 0$. This implies that $\mathfrak{h}_{2} \subset \mathfrak{s o}\left(L_{2}\right)$ is a Berger subalgebra and $\mathfrak{h}_{2} \subset \mathfrak{s p}\left(V_{\overline{1}}\right)$ is a skew-Berger subalgebra.

2. There exists an $R \in \mathcal{R}(\mathfrak{g})_{\overline{0}}$ such that for some $\xi, \delta \in V_{\overline{1}}$ it holds $0 \neq R(\xi, \delta) \in \mathfrak{h}_{2}$. This case is similar to Case 1 and we get the same conclusion.

3. There exists an $R \in \mathcal{R}(\mathfrak{g})_{\overline{1}}$ such that for some $x \in L_{2}$ and $\xi \in V_{\overline{1}}$ it holds $0 \neq R(x, \xi) \in \mathfrak{h}_{2}$. This shows that $\mathfrak{h}_{2} \subset \mathfrak{s o}\left(L_{2}\right)$ is a Berger subalgebra and $\mathfrak{h}_{2} \subset \mathfrak{s p}\left(V_{\overline{1}}\right)$ is a skew-Berger subalgebra.

Thus $L_{2}$ is an irreducible $\mathfrak{h}_{2}$-module or $L_{2}=L \oplus L^{*}$, where $\mathfrak{h}_{2} \subset \mathfrak{g l}(L)$ is irreducible. The same holds for $V_{\overline{1}}$. Next, as in Case a.1, we show that from the irreducibility of $\mathfrak{g} \subset \mathfrak{o s p}(V)$ it follows that $L_{2}=0$ and we get a contradiction.

Case $b . \mathfrak{h}_{1}$ annihilates $V_{\overline{0}}$. This case is analogous to Case a. Note that if $\mathfrak{g}$ is of type I, then in this case $\mathfrak{h}_{1} \subset \mathfrak{s p}\left(V_{\overline{1}}\right)$ and $V_{\overline{1}}$ is a direct sum of $\mathfrak{h}_{1}$-submodules isomorphic to $W_{1}$ and of a 
$\mathfrak{h}_{1}$-trivial submodule. Since $\mathfrak{h}_{1} \subset \mathfrak{s o}\left(W_{1}\right)$, we get that $\mathfrak{h}_{1} \subset \mathfrak{s u}\left(W_{1}\right)$. As above we may consider Cases b.1, b.2, b.3.

We are left with the following case:

Case c. The representations of $\mathfrak{h}_{1}$ and $\mathfrak{h}_{2}$ are diagonal in $V_{\overline{0}} \oplus V_{\overline{1}}$.

We may decompose $V_{\overline{0}}$ as the direct sum $V_{\overline{0}}=L_{1} \oplus L_{2} \oplus L_{3} \oplus L_{4}$ such that $\mathfrak{h}_{1} \oplus \mathfrak{h}_{2}$ annihilates $L_{4}, \mathfrak{h}_{1}$ annihilates $L_{2}, \mathfrak{h}_{2}$ annihilates $L_{1}, L_{1}$ is an $\mathfrak{h}_{1}$-submodule, $L_{2}$ is an $\mathfrak{h}_{2}$-submodule, and $L_{3}$ is an $\mathfrak{h}_{1} \oplus \mathfrak{h}_{2}$-submodule. Let $V_{\overline{0}}=L_{1}^{\prime} \oplus L_{2}^{\prime} \oplus L_{3}^{\prime} \oplus L_{4}^{\prime}$ be the similar decomposition. Since $\mathcal{R}(\mathfrak{g})_{\overline{1}} \neq 0$, we get that $P_{\eta}\left(\operatorname{pr}_{\mathfrak{s o}\left(V_{\overline{0}}\right)} \mathfrak{g}_{\overline{0}}\right) \neq 0$ and $P_{\omega}\left(\operatorname{pr}_{\mathfrak{s p}\left(V_{\overline{1}}\right)} \mathfrak{g}_{\overline{0}}\right) \neq 0$. This shows that if $L_{1} \neq 0$, then $L_{3}=0$. Moreover $L_{2} \neq 0$, since the representation of $\mathfrak{h}_{1}$ is diagonal in $V_{\overline{0}} \oplus V_{\overline{1}}$. Thus either $L_{3}=0$, or $L_{1}=0$ and $L_{2}=0$. Furthermore, if $L_{3} \neq 0$, then $\mathfrak{h}_{1} \oplus \mathfrak{h}_{2} \subset \mathfrak{s o}\left(L_{3}\right)$ is a Berger subalgebra (which is irreducible or $L_{3}$ is of the form $U \oplus U^{*}$ and $\mathfrak{h}_{1} \oplus \mathfrak{h}_{2} \subset \mathfrak{g l}(U)$ is irreducible). Similarly, if $L_{1} \neq 0$ and $L_{2} \neq 0$, then $\mathfrak{h}_{1} \subset \mathfrak{s o}\left(L_{1}\right)$ and $\mathfrak{h}_{2} \subset \mathfrak{s o}\left(L_{2}\right)$ are Berger subalgebras. The same statements we get for $V_{\overline{1}}$ (instead of Berger subalgebras we get skew-Berger subalgebras). If $L_{1} \neq 0, L_{2} \neq 0, L_{1}^{\prime} \neq 0$, and $L_{2}^{\prime} \neq 0$ then we show in the same way as in Case a.1 that the representation is not irreducible. If $L_{1} \neq 0, L_{2} \neq 0$, and $L_{3}^{\prime} \neq 0$, then we show as for the adjoint representations that $\mathcal{R}(\mathfrak{g})_{\overline{1}}=0$. If $L_{3} \neq 0$, and $L_{3}^{\prime} \neq 0$, then either $\mathfrak{h}_{1} \oplus \mathfrak{h}_{2}$ does not appear as a Berger or skew-Berger algebra, or $\mathfrak{h}_{1} \oplus \mathfrak{h}_{2}$ appears only as a reducible Berger and a reducible skew-Berger algebra. In the last case the representation of $\mathfrak{g}$ is not irreducible.

Note that in each case we get a decomposition of $V$ into irreducible $\mathfrak{g}_{\overline{0}}$-modules. Then we ask if such representation of $\mathfrak{g}$ exists, in other words, we should check if the obtained representation of $\mathfrak{g}_{\overline{0}}$ can be extended to an irreducible representation of $\mathfrak{g}$. This can be done by passing to the complex case. Then we may use the theory of representations of the complex simple Lie superalgebras [19, 20, 22, 23, 24, 30, 35, 36, 37]. Any irreducible representation $\mathfrak{g} \subset \mathfrak{g l}(V)$ is the highest-weight representation $V_{\Lambda}$ and the weight $\Lambda$ is given by its labels on the Kac-Dynkin diagram of $\mathfrak{g}$. There is a way to decompose the $\mathfrak{g}_{\overline{0}}$-module $V_{\Lambda}$ into irreducible components. One $\mathfrak{g}_{\overline{0}}$-module $V_{\tilde{\Lambda}}$ is obtained directly from $\Lambda$. Then $V_{\tilde{\Lambda}}$ must coincide with one of the irreducible $\mathfrak{g}_{\overline{0}}$-modules obtained by us. The weight $\tilde{\Lambda}$ defines uniquely $\Lambda$ and we need only to check that $V_{\Lambda}$ consists exactly of the irreducible $\mathfrak{g}_{0}$-modules obtained by us. We will demonstrate this technics in the examples below.

Example 1 Let $\mathfrak{g}$ be the real form of the complex simple Lie superalgebra $F(4)$ with $\mathfrak{g}_{\overline{0}}=$ $\mathfrak{s l}(2, \mathbb{R}) \oplus \mathfrak{s o}(7, \mathbb{R})$ and $\mathfrak{g}_{\overline{1}}=\mathbb{R}^{2} \otimes \Delta$, where $\Delta \simeq \mathbb{R}^{8}$ is the spinor representation of $\mathfrak{s o}(7, \mathbb{R})$.

Case a.1. We have $V_{\overline{0}}=\Delta$ and $V_{\overline{1}}=\mathbb{R}^{2}$. Note that $V_{\overline{0}} \otimes V_{\overline{1}}=\mathfrak{o s p}(8 \mid 2, \mathbb{R})_{\overline{1}}$, hence $\left[V_{\overline{0}}, V_{\overline{1}}\right]=$ $\mathfrak{s l}(2, \mathbb{R}) \oplus \mathfrak{s o}(8, \mathbb{R})$. This shows that the representation of $\mathfrak{g}$ on $\mathbb{R}^{8 \mid 2}$ does not exist.

Case a.2. We consider it after Case a.3.

Case a.3. We have $V_{\overline{0}}=\Delta \oplus L$, where $\mathfrak{s l}(2, \mathbb{R}) \subset \mathfrak{s o}(L)$ is an irreducible Berger subalgebra, and $V_{\overline{1}}=\mathbb{R}^{2}$. As in Case a.1, such representation does not exist. We may prove it also in another way. First since $\mathfrak{s l}(2, \mathbb{R}) \subset \mathfrak{s o}(L)$ is an irreducible Berger algebra, the only possible $L$ are $\mathbb{R}^{3}$ and $\mathbb{R}^{5}$. Passing to the complexification we get that as $\mathfrak{g}_{\overline{0}}=\mathfrak{s l}(2, \mathbb{C}) \oplus \mathfrak{s o}(7, \mathbb{C})$-modules, $V_{\overline{0}}=\mathbb{C}^{8} \oplus L$, where $\mathbb{C}^{8}$ is the spinor representation of $\mathfrak{s o}(7, \mathbb{C})$, L is either $\mathbb{C}^{3}$, or $\mathbb{C}^{5}$, and $V_{\overline{1}}=\Pi \mathbb{C}^{2}$. Note that neither $\mathfrak{g}_{\overline{1}} \otimes \mathbb{C}^{8}$, nor $\mathfrak{g}_{\overline{1}} \otimes \mathbb{C}^{2}$ contain any of the $\mathfrak{g}_{\overline{0}}$-modules $\mathbb{C}^{3}$ and $\mathbb{C}^{5}$. This means that $\mathfrak{g}_{\overline{1}} \cdot\left(\mathbb{C}^{8} \oplus \Pi \mathbb{C}^{2}\right) \subset \mathbb{C}^{8} \oplus \Pi \mathbb{C}^{2}$, i.e. the vector supersubspace $\mathbb{C}^{8} \oplus \Pi \mathbb{C}^{2} \subset V$ is $\mathfrak{g}$-invariant, hence $L=0$. In fact, the method of the decomposition of a $\mathfrak{g}_{\overline{0}}$-module $V$ into irreducible components discussed above is founded on the fact that if $U \subset V$ is an irreducible $\mathfrak{g}_{\overline{0}}$-module, then $\mathfrak{g}_{\overline{1}}$ takes it into some irreducible components of the tensor product $\mathfrak{g}_{\overline{1}} \otimes U$.

Let us show how the above discussed method can be applied to our representations. The information about the irreducible representations of $F(4)$ can be found in [35]. Any irreducible representation (with the highest weight $\Lambda$ ) of $F(4)$ is given by the labels $\left(a_{1}, a_{2}, a_{3}, a_{4}\right)$ on the Kac-Dynkin diagram. Define the following number $b=\frac{1}{3}\left(2 a_{1}-3 a_{2}-4 a_{3}-2 a_{4}\right)$. The labels must satisfy the conditions: $b, a_{2}, a_{3}, a_{4}$ are non-negative integers; if $b=0$, then $a_{1}=\cdots=a_{4}=0$; 
$b \neq 1$; if $b=2$, then $a_{2}=a_{4}=0 ;$ if $b=3$, then $a_{2}=2 a_{4}+1$. The weight $\tilde{\Lambda}$ is given by the labels $\left(b, a_{2}, a_{3}, a_{4}\right)$ on the Dynkin diagram of the Lie algebra $\mathfrak{s l}(2, \mathbb{C}) \oplus \mathfrak{s o}(7, \mathbb{C})$. In our case $\tilde{\Lambda}$ must be one of $(0,0,0,1),(1,0,0,0),(2,0,0,0),(4,0,0,0)$. The first two cases do not satisfy the conditions on the labels. The third case corresponds to the adjoint representation, which is different from our ones. In the second case $V$ contains a $\mathfrak{g}_{\overline{0}}$-module with the highest weight $(3,1,0,0)$, while our representations do not contain such submodule.

Coming back to Case a.2 we get that in this case the representation is given by $b=1, a_{2}=0$, $a_{3}=0$, and $a_{4}=1$. But the representations of $F(4)$ with $b=1$ do not exist.

Case b. Does not appear, since the representation of $\mathfrak{s o}(7, \mathbb{R})$ in $\Delta$ is not unitary.

Case c. Section $[2$ and the papers [5, 17] show that the only representation of $\mathfrak{s l}(2, \mathbb{R}) \oplus \mathfrak{s o}(7, \mathbb{R})$ as a skew-Berger algebra is in the space $\mathbb{R}^{2} \otimes \Delta$, and Lie algebra $\mathfrak{s l}(2, \mathbb{R}) \oplus \mathfrak{s o}(7, \mathbb{R})$ does not appear as the Berger subalgebra of $\mathfrak{s o}(p, q)$. Thus, as we have seen, in this case $\mathcal{R}(\mathfrak{g})_{\overline{1}}=0$.

Example 2 Let $\mathfrak{g}$ be the real form of the complex simple Lie superalgebra osp $(4 \mid 2, \alpha, \mathbb{C})$ with $\mathfrak{g}_{\overline{0}}=\mathfrak{s l}(2, \mathbb{R}) \oplus \mathfrak{s l}(2, \mathbb{R}) \oplus \mathfrak{s l}(2, \mathbb{R})$ and $\mathfrak{g}_{\overline{1}}=\mathbb{R}^{2} \otimes \mathbb{R}^{2} \otimes \mathbb{R}^{2}$. Suppose that $\mathfrak{g} \subset \mathfrak{o s p}(p, q \mid 2 m)=\mathfrak{o s p}(V)$ is an irreducible Berger supersubalgebra. We may consider several cases.

First suppose that the representation of none of the Lie algebras $\mathfrak{s l}(2, \mathbb{R})$ is diagonal in $V_{\overline{0}} \oplus V_{\overline{1}}$. Using the fact that the representation of $\mathfrak{s l}(2, \mathbb{R})$ in $\mathbb{R}^{2}$ is symplectic and the arguments of Case a.1, we get that $V_{\overline{1}}=\mathbb{R}^{2}$ as the $\mathfrak{s l}(2, \mathbb{R})$-module, and $V_{\overline{0}}=\mathbb{R}^{2} \otimes \mathbb{R}^{2}$ as the $\mathfrak{s l}(2, \mathbb{R}) \oplus \mathfrak{s l}(2, \mathbb{R})$ module. To analyze such representations we turn to the complex case. Then we may use the theory of representations of the complex simple Lie superalgebras osp $(4 \mid 2, \alpha, \mathbb{C}$ ] [22]. Any representation of $\mathfrak{o s p}(4 \mid 2, \alpha, \mathbb{C})$ is given by the numbers $\left(a_{2}, a_{1}, a_{3}\right)$ (the labels on the Kac-Dynkin diagram of $\mathfrak{o s p}(4 \mid 2, \alpha, \mathbb{C}))$ such that $a_{2}, a_{3}$ and the number $b=\frac{1}{1+\alpha}\left(2 a_{1}-a_{2}-\alpha a_{3}\right)$ are nonnegative integers. Furthermore, if $b=0$, then $a_{1}=a_{2}=a_{3}=0$; if $b=1$, then $\alpha\left(a_{3}+1\right)=$ $\pm\left(a_{2}+1\right)$. In [22] it is shown that $V$ contains the following $\mathfrak{s l}(2, \mathbb{C}) \oplus \mathfrak{s l}(2, \mathbb{C}) \oplus \mathfrak{s l}(2, \mathbb{C})$ submodules: $\left(b, a_{2}, a_{3}\right),\left(b-1, a_{2} \pm 1, a_{3} \pm 1\right),\left(b-1, a_{2} \pm 1, a_{3} \mp 1\right),\left(b-2, a_{2} \pm 2, a_{3}\right),\left(b-2, a_{2}, a_{3} \pm 2\right)$, $\left(b-2, a_{2}, a_{3}\right),\left(b-3, a_{2} \pm 1, a_{3} \pm 1\right),\left(b-3, a_{2} \pm 1, a_{3} \mp 1\right),\left(b-4, a_{2}, a_{3}\right)$ (the representations are given by the labels on the Dynkin diagram of $\mathfrak{s l}(2, \mathbb{C}) \oplus \mathfrak{s l}(2, \mathbb{C}) \oplus \mathfrak{s l}(2, \mathbb{C}))$. In our case $\left(b, a_{2}, a_{3}\right)$ is one of $(1,0,0),(0,1,0),(0,0,1),(0,1,1),(1,1,0),(1,0,1)$. In the first case $\alpha=1$ and we get the identity representation of $\mathfrak{o s p}(4 \mid 2, \mathbb{R})$; the second, the third and the forth cases are not possible; in the last two cases $V$ contains the representations $(0,2,1)$ and $(0,1,2)$ that give the contradiction. Thus the only possible representation is the identity representation of $\mathfrak{o} \mathfrak{s p}(4 \mid 2, \mathbb{R})$.

Next we suppose that a number of the representation of the Lie algebras $\mathfrak{s l}(2, \mathbb{R})$ are diagonal in $V_{\overline{0}} \oplus V_{\overline{1}}$. By the same arguments as in Cases a.3 and b we show that if $\mathfrak{g}$ is a Berger superalgebra, then it is symmetric.

The representations of the simple Lie superalgebras $\mathfrak{g}$ such that the semi-simple part of $\mathfrak{g}_{\overline{0}}$ is simple can be considered in the same way. The situation becomes simpler, since the representation of $\mathfrak{g}_{\overline{0}}$ is diagonal in $V_{\overline{0}} \oplus V_{\overline{1}}$ (except for the identity representations of $\mathfrak{o s p}(1 \mid 2 m, \mathbb{R}$ ) and $\mathfrak{o s p}(2 \mid 2 m, \mathbb{R}))$. We immediately conclude that $\operatorname{pr}_{\mathfrak{s o}\left(V_{\overline{0}}\right)} \mathfrak{g}_{\overline{0}} \subset \mathfrak{s o}\left(V_{\overline{0}}\right)$ is a Berger subalgebra and $\operatorname{pr}_{\mathfrak{s p}\left(V_{\overline{1}}\right)} \mathfrak{g}_{\overline{0}} \subset \mathfrak{s p}\left(V_{\overline{1}}\right)$ is a skew-Berger subalgebra.

Example 3 Consider the Lie superalgebra $\mathfrak{g}=\mathfrak{p} \mathfrak{e}(n, \mathbb{R})$. Recall that $\mathfrak{g}_{\overline{0}}=\mathfrak{s l}(n, \mathbb{R})$ and $\mathfrak{g}_{\overline{1}}=$ $\odot^{2} \mathbb{R}^{n} \oplus \Lambda^{2} \mathbb{R}^{n *}$. Suppose that $\mathfrak{g} \subset \mathfrak{o s p}(p, q \mid 2 m)=\mathfrak{o} \mathfrak{s p}(V)$ is an irreducible non-symmetric Berger algebra. Then $\operatorname{pr}_{\mathfrak{s o}\left(V_{\overline{0}}\right)} \mathfrak{g}_{\overline{0}} \subset \mathfrak{s o}\left(V_{\overline{0}}\right)$ is a Berger algebra and $\operatorname{pr}_{\mathfrak{s p}\left(V_{\overline{1}}\right)} \mathfrak{g}_{\overline{0}} \subset \mathfrak{s p}\left(V_{\overline{1}}\right)$ is a skew-Berger algebra. Section 2 and the results from [5, 17] show that $V_{\overline{0}}$ and $V_{\overline{1}}$ should be contained in the following list:

$\mathfrak{s l}(n, \mathbb{R}), \mathbb{R}^{n} \oplus \mathbb{R}^{n *}, \odot^{2} \mathbb{R}^{n} \oplus \odot^{2} \mathbb{R}^{n *}, \Lambda^{2} \mathbb{R}^{n} \oplus \Lambda^{2} \mathbb{R}^{n *}$, $\Lambda^{3} \mathbb{R}^{6}(n=6)$, and $\Lambda^{4} \mathbb{R}^{8}(n=8)$. 
To study these representations we turn to the complexification. Suppose, for example, that $V_{\overline{0}}=\mathbb{C}^{n} \oplus \mathbb{C}^{n *}$. The $\mathfrak{g}_{\overline{0}}$-submodule $\mathfrak{g}_{\overline{1}} \cdot \mathbb{C}^{n}$ must coincide with an irreducible component from the list obtaining by the complexification of the above one. We have

$$
\mathfrak{g}_{\overline{1}} \otimes \mathbb{C}^{n}=V_{3 \pi_{1}} \oplus V_{\pi_{1}+\pi_{2}} \oplus V_{\pi_{1}+\pi_{n-2}} \oplus \mathbb{C}^{n *}
$$

Hence, $\mathfrak{g}_{\overline{1}} \cdot \mathbb{C}^{n}=\mathbb{C}^{n *} \subset V_{\overline{1}}$. The tensor product $\mathfrak{g}_{\overline{1}} \otimes \mathbb{C}^{n *}$ does not contain $\mathbb{C}^{n *}$. This means that the vector supersubspace $\mathbb{C}^{n} \oplus \mathbb{C}^{n *}$ (where $\mathbb{C}^{n *} \subset V_{\overline{1}}$ ) of $V$ is $\mathfrak{g}$-invariant and we get a contradiction. All the other representatives from the above list can be considered in a similar way.

Thus we conclude that if $\mathfrak{g}$ is a simple real Lie superalgebra and there exists an irreducible representation $\mathfrak{g} \subset \mathfrak{o} \mathfrak{s p}(p, q \mid 2 m)$ such that $\mathfrak{g}$ is a non-symmetric Berger subalgebra, then this representation is the identity one of $\mathfrak{g}$ and $\mathfrak{g}$ is one of the following Lie algebras with their identity representations: $\mathfrak{o s p}(p, q \mid 2 m), \mathfrak{o s p}(p \mid 2 m, \mathbb{C}), \mathfrak{s u}\left(p_{0}, q_{0} \mid p_{1}, q_{1}\right)$ and $\mathfrak{h o s p}(p, q \mid m)$. These Lie superalgebras with their identity representations are non-symmetric Berger superalgebras, since they contain, respectively, the subalgebras $\mathfrak{s o}(p, q), \mathfrak{s o}(p, \mathbb{C}), \mathfrak{s u}\left(p_{0}, q_{0}\right)$ and $\mathfrak{h o s p}(p, q)$, which are non-symmetric Berger algebras.

Suppose that $\mathfrak{g}$ is a simple real Lie superalgebra and there exists an irreducible representation $\mathfrak{g} \subset \mathfrak{o s p}(p, q \mid 2 m)$ such that $\mathfrak{g} \oplus \mathfrak{z}$ is a non-symmetric Berger supersubalgebra, where $\mathfrak{z}$ is a supersubalgebra of $\mathfrak{o s p}(p, q \mid 2 m)$ commuting with $\mathfrak{g}$. Since $\mathfrak{g}$ is not contained in $\mathfrak{q}(n, \mathbb{R})$, i.e. it does not commute with an odd complex structure, by the Schur Lemma for representations of Lie superalgebras, $\mathfrak{z}$ is either $\mathbb{R} J$, where $J$ is an even complex structure, or $\mathfrak{z}=\mathfrak{s p}(1)$, i.e. $\mathfrak{z}$ is spanned by an even quaternionic structure $J_{1}, J_{2}, J_{3}$. From Section 2 it follows that if $\mathfrak{h} \subset \mathfrak{s o}(p, q)$ is an irreducible subalgebra, then if $\mathfrak{h} \oplus \mathfrak{z}$ is a non-symmetric Berger algebra, then $\mathfrak{h}$ is a non-symmetric Berger algebra. Similarly, from [17] it follows that if $\mathfrak{h} \subset \mathfrak{s p}(2 m, \mathbb{R})$ is an irreducible subalgebra then if $\mathfrak{h} \oplus \mathfrak{z}$ is a skew-Berger algebra, then $\mathfrak{h}$ is a skew-Berger algebra. The same note holds for representations in $U \oplus U^{*}$, where $\mathfrak{h} \subset \mathfrak{s l}(U)$ is irreducible. This shows that the above method can be applied also to irreducible subalgebras $\mathfrak{g} \oplus \mathfrak{z} \subset \mathfrak{o s p}(p, q \mid 2 m)$, where $\mathfrak{g}$ is simple. We obtain the identity representations of $\mathfrak{u}\left(p_{0}, q_{0} \mid p_{1}, q_{1}\right), \mathfrak{h o s} \mathfrak{p}(p, q \mid m) \oplus \mathbb{R} J$ and $\mathfrak{h o s p}(p, q \mid m) \oplus \mathfrak{s p}(1)$. Since $\mathfrak{u}\left(p_{0}, q_{0} \mid p_{1}, q_{1}\right)$ contains $\mathfrak{u}\left(p_{0}, q_{0}\right)$, it is a non-symmetric Berger superalgebra. Since

$$
\mathcal{R}(\mathfrak{s p}(p, q) \oplus \mathbb{R} J)=\mathcal{R}(\mathfrak{s} \mathfrak{p}(p, q)), \quad \overline{\mathcal{R}}(\mathfrak{s o}(m, \mathbb{H}) \oplus \mathbb{R} J)=\overline{\mathcal{R}}(\mathfrak{s o}(m, \mathbb{H})),
$$

and $J$ acts diagonally in $V_{\overline{0}} \oplus V_{\overline{1}}$, we get that

$$
\mathcal{R}(\mathfrak{h o s p}(p, q \mid m) \oplus \mathbb{R} J)=\mathcal{R}(\mathfrak{h o s p}(p, q \mid m)),
$$

i.e. $\mathfrak{h o s p}(p, q \mid m) \oplus \mathbb{R} J$ is not a Berger superalgebra. Generalizing the curvature tensor of the quaternionic projective space (with indefinite metric) [2], we define the curvature tensor $R \in \mathcal{R}(\mathfrak{h o s p}(p, q \mid m) \oplus \mathfrak{s p}(1))$ by

$$
R(X, Y)=\frac{1}{2} \sum_{\alpha=1}^{3} g\left(J_{\alpha} X, Y\right) J_{\alpha}-\frac{1}{4}\left(X \wedge Y+\sum_{\alpha=1}^{3} J_{\alpha} X \wedge J_{\alpha} Y\right),
$$

where $X, Y \in V$. The restriction of $R$ to $\Lambda^{2} \mathbb{R}^{4 p, 4 q}$ coincides with the curvature tensor of the quaternionic projective space, its image is not contained in $\mathfrak{s p}(p, q)$. This shows that $R \notin \mathcal{R}(\mathfrak{h o s p}(p, q \mid m))$. Thus,

$$
\mathcal{R}(\mathfrak{h o s p}(p, q \mid m)) \neq \mathcal{R}(\mathfrak{h o s p}(p, q \mid m) \oplus \mathfrak{s p}(1))
$$

and $\mathfrak{h o s p}(p, q \mid m) \oplus \mathfrak{s p}(1)$ is a non-symmetric Berger superalgebra. 
Let now $\mathfrak{g}^{1} \subset \mathfrak{g l}\left(V^{1}\right)$ and $\mathfrak{g}^{2} \subset \mathfrak{g l}\left(V^{2}\right)$ be two irreducible real supersubalgebras. Consider the tensor product of these representations $\mathfrak{g}=\mathfrak{g}^{1} \oplus \mathfrak{g}^{2} \subset \mathfrak{g l}\left(V^{1} \otimes V^{2}\right)=\mathfrak{g l}(V)$. Suppose that $\mathfrak{g} \subset \mathfrak{o s p}(V)$. Note that if the real vector superspaces $V^{1}$ and $V^{2}$ admit complex structures commuting, respectively, with the elements of $\mathfrak{g}^{1}$ and $\mathfrak{g}^{2}$, then the representation of $\mathfrak{g}=\mathfrak{g}^{1} \oplus \mathfrak{g}^{2}$ in $V^{1} \otimes V^{2}$ is reducible and we consider its representation in $V^{1} \otimes_{\mathbb{C}} V^{2}$.

For the even and odd parts of $V^{1} \otimes V^{2}$ we have

$$
\left(V^{1} \otimes V^{2}\right)_{\overline{0}}=V_{\overline{0}}^{1} \otimes V_{\overline{0}}^{2} \oplus V_{\overline{1}}^{1} \otimes V_{\overline{1}}^{2}, \quad\left(V^{1} \otimes V^{2}\right)_{\overline{1}}=V_{\overline{0}}^{1} \otimes V_{\overline{1}}^{2} \oplus V_{\overline{1}}^{1} \otimes V_{\overline{0}}^{2} .
$$

This shows that if the even and odd parts of both $V^{1}$ and $V^{2}$ are non-trivial, then the representation of $\mathfrak{g}_{\overline{0}}$ is diagonal in $\left(V^{1} \otimes V^{2}\right)_{\overline{0}} \oplus\left(V^{1} \otimes V^{2}\right)_{\overline{1}}$. Consequently, $\operatorname{pr}_{\mathfrak{s o}\left(\left(V^{1} \otimes V^{2}\right)_{\overline{0}}\right)} \mathfrak{g}_{\overline{0}}$ is a Berger algebra and $\operatorname{pr}_{\mathfrak{s p}\left(\left(V^{1} \otimes V^{2}\right)_{\overline{1}}\right)} \mathfrak{g}_{\overline{0}}$ is a skew-Berger algebra. Using the arguments as above, it is easy to see that if $\mathfrak{g}$ is a Berger superalgebra, then it is symmetric. The same works for the tensor product of several representations (we may assume that $\mathfrak{g}^{2}$ is a direct some of simple Lie superalgebras and $V^{2}$ is a tensor products of irreducible representations of these Lie superalgebras).

Next, we assume that the even and odd parts of $V^{1}$ are non-trivial and $V^{2}$ is either purely even or purely odd. If $V^{2}$ is purely odd, then $V^{1} \otimes V^{2}=\Pi V^{1} \otimes \Pi V^{2}$, where $\Pi V^{2}$ is purely even. Thus we may assume that $V^{2}$ is purely even, i.e. $V^{2}$ is a usual vector space. Since $\mathfrak{g}=\mathfrak{g}^{1} \oplus \mathfrak{g}^{2} \subset \mathfrak{o} \mathfrak{s} \mathfrak{p}\left(V^{1} \otimes V^{2}\right)$, we get that either $\mathfrak{g}^{1} \subset \mathfrak{o} \mathfrak{s p}\left(V^{1}\right)$ and $\mathfrak{g}^{2} \subset \mathfrak{s o}\left(V^{2}\right)$, or $\mathfrak{g}^{1} \subset \mathfrak{o} \mathfrak{s p}{ }^{s k}\left(V^{1}\right)$ and $\mathfrak{g}^{2} \subset \mathfrak{s p}\left(V^{2}\right)$.

Let $V^{1}$ and $V^{2}$ be a complex vector superspace and a complex vector space, respectively, and let $V=V^{1} \otimes V^{2}$. Let $g_{1}$ be a supersymmetric bilinear forms on $V^{1}$ and $g_{2}$ be a symmetric bilinear form on $V^{2}$ From the results of [27] it follows that

$$
\mathcal{R}\left(\mathfrak{s l}\left(V^{1}\right) \oplus \mathfrak{s l}\left(V^{2}\right) \oplus \mathbb{C}\right) \simeq V^{*} \otimes V^{*}
$$

Any $\tau \in V^{*} \otimes V^{*}$ defines the curvature tensor $R_{\tau}$ by

$$
R_{\tau}\left(x_{1} \otimes x_{2}, u_{1} \otimes u_{2}\right)=A\left(x_{1} \otimes x_{2}, u_{1} \otimes u_{2}\right)+B\left(x_{1} \otimes x_{2}, u_{1} \otimes u_{2}\right),
$$

where $A\left(x_{1} \otimes x_{2}, u_{1} \otimes u_{2}\right) \in \mathfrak{s l}\left(V^{1}\right) \oplus \mathbb{C}, B\left(x_{1} \otimes x_{2}, u_{1} \otimes u_{2}\right) \in \mathfrak{s l}\left(V^{2}\right) \oplus \mathbb{C}$, and for $v_{1} \in V^{1}$ and $v_{2} \in V^{2}$ we have

$$
\begin{gathered}
A\left(x_{1} \otimes x_{2}, u_{1} \otimes u_{2}\right) v_{1}=(-1)^{\left|v_{1}\right|\left|u_{1}\right|} \tau\left(x_{1}, x_{2}, v_{1}, u_{2}\right) u_{1}-(-1)^{\left(\left|v_{1}\right|+\left|u_{1}\right|\right)\left|x_{1}\right|} \tau\left(u_{1}, u_{2}, v_{1}, x_{2}\right) x_{1}, \\
B\left(x_{1} \otimes x_{2}, u_{1} \otimes u_{2}\right) v_{2}=\tau\left(x_{1}, x_{2}, u_{1}, v_{2}\right) u_{2}-(-1)^{\left|u_{1}\right|\left|x_{1}\right|} \tau\left(u_{1}, u_{2}, x_{1}, v_{2}\right) x_{2} .
\end{gathered}
$$

In particular,

$$
\operatorname{tr}\left(B\left(x_{1} \otimes x_{2}, u_{1} \otimes u_{2}\right)\right)=\left(\tau\left(x_{1}, x_{2}, u_{1}, u_{2}\right)-(-1)^{\left|u_{1}\right|\left|x_{1}\right|} \tau\left(u_{1}, u_{2}, x_{1}, x_{2}\right)\right) .
$$

If $q>1$, then by the same arguments as in [29, 16] it can be shown that if $R_{\tau} \in \mathcal{R}\left(\mathfrak{o s p}\left(V^{1}\right) \oplus\right.$ $\left.\mathfrak{s o}\left(V^{2}\right)\right) \subset \mathcal{R}\left(\mathfrak{s l}\left(V^{1}\right) \oplus \mathfrak{s l}\left(V^{2}\right) \oplus \mathbb{C}\right)$ then it is given by $\tau \in \odot^{2} V^{*}$ such that

$$
\tau\left(x_{1}, x_{2}, u_{1}, u_{2}\right)=c g_{1}\left(x_{1}, u_{1}\right) g_{2}\left(x_{2}, u_{2}\right),
$$

where $c \in \mathbb{C}$. Hence,

$$
\mathcal{R}\left(\mathfrak{o s p}\left(V^{1}\right) \oplus \mathfrak{s o}\left(V^{2}\right)\right)=\mathcal{R}\left(\mathfrak{o s p}\left(V^{1}\right) \oplus \mathfrak{s o}\left(V^{2}\right)\right)_{\overline{0}}
$$

is one-dimensional. Thus $\mathfrak{o} \mathfrak{s p}\left(V^{1}\right) \oplus \mathfrak{s o}\left(V^{2}\right) \subset \mathfrak{o} \mathfrak{s p}\left(V^{1} \otimes V^{2}\right)$ is a symmetric Berger superalgebra and $\mathcal{R}(\mathfrak{g})=0$ for any proper supersubalgebra $\mathfrak{g} \subset \mathfrak{o s p}\left(V^{1}\right) \oplus \mathfrak{s o}\left(V^{2}\right) \subset \mathfrak{o s p}\left(V^{1} \otimes V^{2}\right)$. Similarly, if $g_{1}$ is a super-skew-symmetric bilinear forms on $V^{1}$ and $g_{2}$ is a symplectic form on $V^{2}$, then

$$
\mathcal{R}\left(\mathfrak{o s p} \mathfrak{p}^{s k}\left(V^{1}\right) \oplus \mathfrak{s p}\left(V^{2}\right)\right)=\mathcal{R}\left(\mathfrak{o} \mathfrak{s p}^{s k}\left(V^{1}\right) \oplus \mathfrak{s p}\left(V^{2}\right)\right)_{\overline{0}}=\mathbb{C} R_{\tau}
$$


where

$$
\tau\left(x_{1}, x_{2}, u_{1}, u_{2}\right)=g_{1}\left(x_{1}, u_{1}\right) g_{2}\left(x_{2}, u_{2}\right) .
$$

The same holds if $V^{1}$ and $V^{2}$ are real.

Thus we are left with the cases $\mathfrak{g}^{2}=\mathfrak{s l}(2, \mathbb{R})$ and $\mathfrak{g}^{2}=\mathfrak{s l}(2, \mathbb{C})$ (in the last case $\mathfrak{g}^{1} \subset \mathfrak{o} \mathfrak{s p}^{s k}\left(V^{1}\right)$ admits a complex structure and we consider the representation of $\mathfrak{g}^{1} \oplus \mathfrak{g}^{2}$ in $\left.V^{1} \otimes_{\mathbb{C}} V^{2}\right)$. Suppose that $\mathfrak{g}^{2}=\mathfrak{s l}(2, \mathbb{R})$. We have $\mathfrak{g}^{1} \subset \mathfrak{o s p}^{s k}\left(V^{1}\right)$. Since there are no reductive Lie algebras $\mathfrak{h}$ such that $\mathfrak{h} \oplus \mathfrak{s l}(2, \mathbb{R})$ appears both as a Berger subalgebra of $\mathfrak{s o}(p, q)$ and as a skew-Berger subalgebra of $\mathfrak{s p}(2 m, \mathbb{C})$, there are no ideal in $\mathfrak{g}_{\overline{0}}^{1}$ that acts diagonally in $V_{\overline{0}}^{1} \oplus V_{\overline{1}}^{1}$. Hence $\mathfrak{g}^{1} \subset \mathfrak{o s} \mathfrak{p}^{s k}\left(V^{1}\right)$ is the identity representation of the Lie superalgebra $\mathfrak{g}^{1}$ (this representation must exist). Section 2. the results of [17] and the condition $\mathcal{R}(\mathfrak{g})_{\overline{1}} \neq 0$ imply $\mathfrak{g}^{1}=\mathfrak{o s p}^{s k}\left(V^{1}\right)=\mathfrak{o s p}^{s k}(2 m \mid r, s)$. Similarly, if $\mathfrak{g}^{2}=\mathfrak{s l}(2, \mathbb{C})$, then $\mathfrak{g}^{1}=\mathfrak{o} \mathfrak{s p}^{s k}(2 m \mid r, \mathbb{C})$. We get the following two algebras:

$$
\mathfrak{o s p}^{s k}(2 m \mid r, s) \oplus \mathfrak{s l}(2, \mathbb{R}) \subset \mathfrak{o s p}\left(\mathbb{R}^{2 m \mid r, s} \otimes \mathbb{R}^{2}\right), \mathfrak{o} \mathfrak{s p}^{s k}(2 m \mid r, \mathbb{C}) \oplus \mathfrak{s l}(2, \mathbb{C}) \subset \mathfrak{o s p}\left(\mathbb{C}^{2 m \mid r} \otimes \mathbb{C}^{2}\right) .
$$

The second representation is the complexification of the identity representation of $\mathfrak{h o s p}(r, r \mid m) \oplus$ $\mathfrak{s p}(1)$, hence the second representation gives us a non-symmetric Berger superalgebra. The complexification of the first representation is the second one, hence the first representation gives us a non-symmetric Berger superalgebra.

The theorem is proved.

Acknowledgments. I am grateful to D. V. Alekseevsky for useful discussions on the topic of this paper.

\section{References}

[1] R. Ahl Laamara, A. Belhaj, L. B. Drissi, E. H. Saidi, On local Calabi-Yau supermanifolds and their mirrors. J. Phys. A 39 (2006), no. 20, 5965-5977.

[2] D. V. Alekseevsky, Riemannian manifolds with exceptional holonomy groups. Functional Analysis and Its Applications, 2 (1968), no. 2, 97-105.

[3] M. Asorey, P. M. Lavrov, Fedosov and Riemannian supermanifolds. J. Math. Phys. 50 (2009), no. 1, 013530, 16 pp.

[4] M. Berger, Sur les groupers d'holonomie des variétés àconnexion affine et des variétés riemanniennes. Bull. Soc. Math. France 83 (1955), 279-330.

[5] M. Berger, Les espace symétriques non compacts. Ann. Sci. école Norm. Sup. V.74 (1957), 85-177.

[6] A. L. Besse, Einstein manifolds. Springer-Verlag, Berlin-Heidelberg-New York, 1987.

[7] P. A. Blaga, Riemannian connections on supermanifolds: a coordinate-free approach. Mathematica 47(70) (2005), no. 1, 27-34.

[8] R. Bryant, Classical, exceptional, and exotic holonomies: a status report. Actes de la Table Ronde de Géométrie Différentielle (Luminy, 1992). Semin. Congr., 1, Soc. Math. France, Paris (1996), 93-165.

[9] D. J. Cirilo-Lombardo, Riemannian superspaces, exact solutions and the geometrical meaning of the field localization. Internat. J. Theoret. Phys. 47 (2008), no. 11, 3015-3028.

[10] V. Cortés, A new construction of homogeneous quaternionic manifolds and related geometric structures. Mem. Amer. Math. Soc. 147 (2000), no. 700, viii+63 pp. 
[11] P. Deligne, J. W. Morgan, Notes on supersymmetry (following Joseph Bernstein), Quantum Fields and Strings: A Course for Mathematicians. Vols. 1,2 (Princeton, NJ, 1996/1997), 41-97. American Mathematical Society, Providence, R.I., 1999.

[12] A. Galaev, T. Leistner, Recent developments in pseudo-Riemannian holonomy theory. Handbook of pseudo-Riemannian geometry and supersymmetry, 581627, IRMA Lect. Math. Theor. Phys., 16, Eur. Math. Soc., Zürich, 2010.

[13] A. Galaev, T. Leistner, Holonomy groups of Lorentzian manifolds: classification, examples, and applications. Recent developments in pseudo-Riemannian geometry, ESI Lect. Math. Phys., Eur. Math. Soc., Zürich (2008), 53-96.

[14] A. S. Galaev, One component of the curvature tensor of a Lorentzian manifold. J. Geom. Phys., 60 (2010), 962-971.

[15] A. S. Galaev, Holonomy of supermanifolds. Abhandlungen aus dem Mathematischen Seminar der Universitat Hamburg, Volume 79, (2009), Issue 1, 47-78.

[16] A. S. Galaev, Irreducible complex skew-Berger algebras. Differential Geom. Appl. 27 (2009), no. $6,743-754$.

[17] A. S. Galaev, Irreducible holonomy algebras of Riemannian odd supermanifolds. Lobachevskii J. Math. 32 (2011), no. 2, 16 pp. (to appear), currently avilable as arXiv:1101.0495.

[18] O. Goertsches, Riemannian Supergeometry. Math. Z. 260 (2008), no. 3, 557-593.

[19] J. P. Hurni, B. Morel, Irreducible representations of superalgebras of type II. J. Math. Phys. 23 (1982), no. 12, 2236-2243.

[20] J. P. Hurni, B. Morel, Irreducible representations of $\mathfrak{s u}(m \mid n)$. J. Math. Phys. 24 (1983), $157-163$.

[21] D. Joyce, Riemannian holonomy groups and calibrated geometry. Oxford University Press (2007).

[22] J. Van der Jeugt, Irreducible representations of the exceptional Lie superalgebras $D(2,1 ; \alpha)$. J. Math. Phys. 26 (1985), no. 5, 913-924.

[23] V. G. Kac, Lie superalgebras. Adv. Math., 26 (1977), 8-96.

[24] V. G. Kac, Representations of classical Lie superalgebras. Lectures Notes in Mathematics 676, Springer-Verlag, Berlin (1978).

[25] T. Leistner, On the classification of Lorentzian holonomy groups. J. Differential Geom. 76 (2007), no. 3, 423-484.

[26] D. A. Leites, Introduction to the theory of supermanifolds. Russian Math. Surveys, 35 (1980), no. 1, 1-64.

[27] D. A. Leites, E. Poletaeva, V. Serganova, On Einstein equations on manifolds and supermanifolds. J. Nonlinear Math. Phys. 9 (2002), no. 4, 394-425.

[28] Yu. I. Manin, Gauge Field Theory and Complex Geometry, Grundlehren 289 (1988), Springer Verlag.

[29] S. Merkulov, L. Schwachhöfer Classification of irreducible holonomies of torsion-free affine connections. Ann. Math. 150 (1999), 77-149. 
[30] B. Morel, A. Sciarrino, P. Sorba, Representations of $\mathfrak{o s p}(M \mid 2 n)$ and Young supertableaux. J. Phys. A: Math. Gen. 18 (1985), 1597-1613.

[31] M. Parker, Classification of real simple Lie superalgebras of classical type. J. Math. Phys. 21 (1980), no. 4, 689-697.

[32] E. Poletaeva, Analogues of Riemann tensors for the odd metric on supermanifolds. Acta Appl. Math. 31 (1993), no. 2, 137-169.

[33] M. Roček, N. Wadhwa, On Calabi-Yau supermanifolds. Adv. Theor. Math. Phys. 9 (2005), no. $2,315-320$.

[34] L. J. Schwachhöfer, Connections with irreducible holonomy representations. Adv. Math. 160 (2001), no. 1, 1-80.

[35] A. Sciarrino, P. Sorba, Representations of the superalgebra F(4) and Young supertableaux. J. Phys. A: Math. Gen. 19 (1986), 2241-2248.

[36] A. Sciarrino, P. Sorba, Representations of the Lie superalgebra G(3). Proceedings of the XVth ICGTMP, Philadelphie (1986); World Scientific, Singapore (1987).

[37] L. Frappat, A. Sciarrino, P. Sorba, Dictionary on Lie algebras and superalgebras. Academic Press, Inc., San Diego, CA, 2000. xxii+410 pp.

[38] V. V. Serganova, Classification of simple real Lie superalgebras and symmetric superspaces. Funktsional Anal. Appl. 17 (1983), no. 3, 200-207.

[39] V. S. Varadarajan, Supersymmetry for Mathematicians: An Introduction. American Mathematical Society, Courant ecture notes, Vol. 11, 2004.

[40] H. Wu, On the de Rham decomposition theorem. Illinois J. Math. 8 (1964), 291311.

[41] C. Zhou, On Ricci flat supermanifolds. J. High Energy Phys. 2005, no. 2, 004, 10 pp. 